\title{
Ribosomal DNA methylation as stable biomarkers for detection of
}

\author{
cancer in plasma \\ Xianglin Zhang, Huan Fang, Wei Zhang, Bixi Zhong, Yanda Li, Xiaowo Wang* \\ Department of Automation, Center for Synthetic and Systems Biology, Tsinghua University; \\ Ministry of Education Key Laboratory of Bioinformatics; Bioinformatics Division, BNRIST, \\ Beijing 100084, China \\ *Corresponding author: xwwang@tsinghua.edu.cn
}

\begin{abstract}
Background

Recently, liquid biopsy for cancer detection has pursued great progress. However, there are still a lack of high quality markers. It is a challenge to detect cancer stably and accurately in plasma cell free DNA (cfDNA), when the ratio of cancer signal is low. Repetitive genes or elements may improve the robustness of signals. In this study, we focused on ribosomal DNA which repeats hundreds of times in human diploid genome and investigated performances for cancer detection in plasma.
\end{abstract}

\section{Results}

We collected bisulfite sequencing samples including normal tissues and 4 cancer types and found that intergenic spacer (IGS) of rDNA has high methylation levels and low variation in normal tissues and plasma. Strikingly, IGS of rDNA shows significant hypo-methylation in tumors compared with normal tissues. Further, we collected plasma bisulfite sequencing data from 224 healthy subjects and cancer patients. Means of AUC in testing set were 0.96 (liver cancer), 0.94 (lung cancer and), 0.92 (colon cancer) with classifiers using only $10 \mathrm{CpG}$ sites. Due to the feature of high copy number, when liver cancer plasma WGBS was down-sampled to 10 million raw reads $(0.25 \times$ whole genome coverage), the prediction performance decreased only a bit (mean $\mathrm{AUC}=0.93$ ). Finally, methylation of rDNA could also be used for monitor cancer progression and treatment.

\section{Conclusion}

Taken together, we provided the high-resolution map of rDNA methylation in tumors and supported that methylation of rDNA was a competitive and robust marker for detecting cancer and monitoring cancer progression in plasma.

Key words: ribosomal DNA, liquid biopsy, biomarker, cancer detection, DNA methylation, cfDNA, liver cancer, colon cancer, lung cancer

\section{Background}

Nowadays, liquid biopsy for cancer managements including cancer detection, monitoring and treatment selection develops quickly due to the improvement of genomic and molecular methods, as well as its advantages of non-invasion and overcoming the limitation of tumor heterogeneity compared with traditional tissue biopsy [1-3]. In liquid biopsy, cell-free DNA (cfDNA) becomes one of the most widely studied analysts because of its stability, abundance and potential applications in precision oncology[2]. cfDNA can be released from cells into body fluids through apoptosis, necrosis and active secretion[2, 3]. In the same way, circulating tumor DNA (ctDNA) is released 
from malignant tumors during the progress of cancer. Identifying ctDNA from other cfDNA with tumor DNA-specific features is the principle of cancer detection. Previous studies showed that point mutations, copy number alterations, structural variants, methylation changes, nucleosomes positioning changes and fragment length differences reflected by tumor DNA can be used as diagnostic markers[2]. Among these markers, aberrant DNA methylation provides indispensable information for cancer managements and have been widely studied [2, 4-6].

Bisulfite conversion sequencing is considered as "gold standard" of methylation sequencing[6] and have been widely used to investigate DNA methylation patterns in single base-pair resolution[7]. Whole genome bisulfite sequencing (WGBS) and reduced representation bisulfite sequencing (RRBS) are two common bisulfite conversion-based technologies. WGBS is fairly accurate, reproducible to obtain methylation profiles of whole genome, but is costly and low coverage[7]. RRBS is relatively cost-effective, but covers only $10 \%$ of whole genome and is GC-biased[7]. They have been used for cfDNA researches in liver cancer, lung cancer and colon cancer[8,9].

Finding accurate DNA methylation markers is a key for cancer detection [10-12]. When there is low tumor burden in plasma, bisulfite sequencing of cfDNA from several milliliter plasma might produce unstable results [2]. However, there are some repeat units with high copy number in human genome, and selecting them as biomarkers may enhance the robustness of signals and improve cancer detection performances. In this study, we paid attention to human ribosomal DNA (rDNA) repeat unit, which repeats hundreds of times in diploid human genome.

Human rDNA clusters on the short arms of the five acrocentric chromosomes (chromosomes 13, 14, $15,21,22$ ), and contains a 13-kb transcribed region and a 30-kb intergenic spacer (IGS). Transcribed region encodes $18 \mathrm{~S}, 5.8 \mathrm{~S}$ and $28 \mathrm{~S}$ ribosomal RNA. IGS contains multiple mobile elements, transcription factor binding sites like TP53 binding sites and sequences similar to scaffold attachment regions, which indicates IGS may play a role in enhancement of transcription, recombination and chromosome organization[13]. Recently, a study identified 49 conserved regions in IGS through comparative genome in multiple species[14] and revealed the regulation potentials of IGS.

rDNA is the fundamental genomic element to form nucleolus where ribosomes assemble together[15, 16] and its transcriptional RNA product - ribosomal RNA is indispensable parts of ribosome, which suggests rDNA stability is vital for normal biology processes. Copy number variation of rDNA balances rDNA dosage [17]and is associated with the expression of several functional coherent gene sets[18]. rDNA copy number amplification or deletion is related to tumor genetic context, nucleolus activity, proliferation and protein activity in different cancer types[1922]. The expression of rDNA also shows aberrant patterns in prostate cancer, cervical cancer, breast cancer and myelodysplastic syndromes[23-26]. Overexpression of pre-45S ribosomal RNA promotes colon cancer and is associated with poor survival of colorectal patients[27].

Epigenetic regulation is an important mechanism of keeping rDNA stable [28-30] and its disruption is highly related with aging and cancer[30-33]. Researchers found that transcribed region of rDNA is un-methylated, while IGS is highly methylated, and relatively sharp boundaries are formed between two regions in blood cells[34]. In contrast, IGS becomes partially de-methylated in lung cancer[35] and the promoters or transcribed domain of rDNA show hyper-methylation in tumor tissues of breast cancer, ovarian cancer, endometrial carcinoma and colorectal cancer [36-40]. Hyper-methylation in rDNA locus predict longer survival in ovarian cancer, endometrial carcinoma[38, 39]. In addition, histone modification analysis shows that, liver cancer cells have 
distinct epigenetic pattern compared with normal liver cells[41]. Previous study has integrated ChIP-seq, DNase-seq, MNase-seq and RNA-seq data to study the epigenetic and transcriptional patterns of rDNA in several cell lines[42], however, there is no methylation map of rDNA repeat unit including both transcribed region and IGS in single $\mathrm{CpG}$ resolution, and there is no study focusing on the potential usage of rDNA methylation as cancer detection markers in plasma.

In present study, we examined methylation pattern of each $\mathrm{CpG}$ site in rDNA for tumors and normal tissues with WGBS and RRBS data and found that IGS of rDNA has high methylation level and low variation in normal tissues and plasma samples, but shows hypo-methylation in at least 4 cancer types compared with normal tissues. Finally, we supported that aberrant methylation of $\mathrm{CpG}$ sites in rDNA could be used as competitive and robust markers for cancer detection and monitoring cancer progression in plasma.

\section{Results}

\section{Aberrant methylation of rDNA in malignant tumors}

In adult human tissues, the most abundant DNA methylation occurs in cytosine that belongs to cytosine-phosphate-guanine $(\mathrm{CpG})$ dinucleotides and is crucial to various biological processes[43, 44]. Therefore, we only focused on the methylation of $\mathrm{CpG}$ sites on rDNA. The reference of human rDNA repeated unit contains $3288 \mathrm{CpG}$ sites in total. In order to coherently describe regions on rDNA, we defined 5 zones according to methylation patterns (see Methods) where Zone 1 occupies rDNA 1-15455 base pairs (bps); Zone 2 occupies 15456-27705bps; Zone 3 occupies 2770629080bps; Zone 4 occupies 29081-41755bps; Zone 5 occupies 41756-42999bps (Fig. 1A). Zone 1 is composed of transcribed region and transcription terminator region which is downstream of 3 ' external transcribed spacer (ETS). Zone 3 corresponds to the peak of H3K4me1/2/3 and H3K9ac within IGS reported by previous studies[41, 42]. Zone 5 overlaps with rDNA promoter region. Zone 2 and Zone 4 locate within IGS. The distribution of CpG sites in rDNA is not uniform. Analysis of CpG density in rDNA indicated that Zone 1 and Zone 5 have higher $\mathrm{CpG}$ density than other regions in rDNA (Fig. 1B).

First, we investigated the general methylation patterns in 45 tumor samples and 41 normal tissue samples using WGBS or RRBS data collected from several studies (see Methods). In normal tissues, Zone 1, 3, 5 have low methylation levels and higher variations, while Zone 2, 4 are relatively highly methylated and less variable (Fig. C, Additional file 1: Fig. S1B). Two trends of methylation pattern changes were observed in malignant tumors. Zone 1, 3, 5 has higher methylation in tumors than normal tissues (Fig. 1C). Zone 2 and 4 within IGS show hypo-methylation in tumors. Further, methylation variations in Zone 2 and 4 increase in tumors (Additional file 1: Fig. S1C). There are more $\mathrm{CpG}$ sites that show significantly hypo-methylated than these are significantly hypermethylated (Fig. 1D).

In addition, Correlation between methylation of $\mathrm{CpG}$ sites along rDNA suggests that the $\mathrm{CpG}$ sites can be grouped into two categories. CpG sites of Category 1 and Category 2 locate at Zone 1, 3, 5 and Zone 2, 4 respectively. The methylation level of $\mathrm{CpG}$ sites has high correlations within the same category but low correlations between different categories. Principal component analysis of correlation matrix shows consistent results of two categories (Fig. 1F).

Above all, through comparison of rDNA's methylation in normal tissues and tumors, we showed distinct methylation pattern in tumors that might be used as biomarkers of cancer detection. 
bioRxiv preprint doi: https://doi org/101101/651497; this version posted May 29,2019 . The copyright holder for this preprint (which was not certified by peer review) is the author/funder, who has granted bioRxiv a license to display the preprint in perpetuity. It is made available under aCC-BY-NC-ND 4.0 International license.

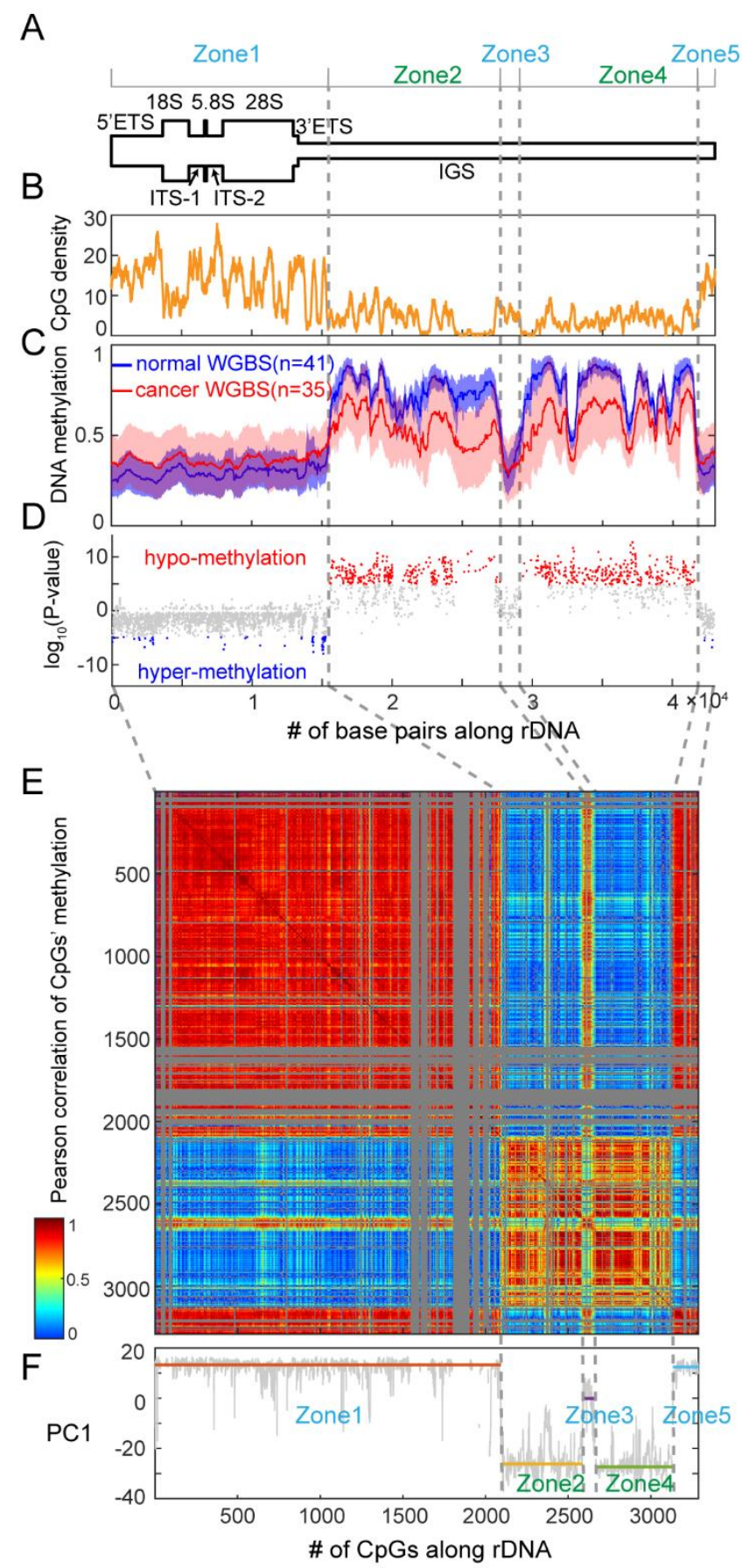

Figure 1 Methylation patterns of tumors and normal tissues

(A) A schematic representation of human $r D N A$ repeat unit. ETS: external transcribed spacer; ITS: internal transcribed spacer; IGS: intergenic spacer. Zones are labeled on the top. (B) Plot of $C p G$ dinucleotides'density along reference sequences. The vertical axis shows the number of CpG dinucleotides per 100 bps. (C) Plots of methylation levels for normal tissues and tumors. The lines indicate the mean methylation levels of tumors and normal tissues. Scopes of plots show the methylation intervals of \pm 1 standard derivation. (D) Scatter plot of differential significance between tumors and normal tissues for each $C p G$ along rDNA. Logarithms of $P$ values of Wilcoxson test are shown as ordinate. Positive values mean hypo-methylation in tumors; negative values mean hyper-methylation in tumors. $C p G$ sites of which Bonferroni adjusted $P$ values are 
bioRxiv preprint doi: https://doi.org/101101/651497; this version posted May 29, 2019. The copyright holder for this preprint (which was not certified by peer review) is the author/funder, who has granted bioRxiv a license to display the preprint in perpetuity. It is made available under aCC-BY-NC-ND 4.0 International license.

smaller than 0.05 are colored as red (hypo-methylation in cancer) and blue (hyper-methylation in cancer). (E) Heat map of correlation matrix between CpG sites along rDNA. Correlation scores of $C p G$ sites with low coverage are labeled as grey. grey dashed lines shows the correspondence between positions and ranked numbers of $C p G$ dinucleotides. (F) Plot of principal component 1 (PC1) of correlation matrix. For each zone, median of PC1 values is drawn as horizontal line.

\section{Hypo-methylation of IGS is a common feature in different cancer types}

In order to further interrogate the features of aberrant methylation in tumors, we divided samples into different subsets and investigated their methylation profiles separately. First, we examined methylation heterogeneity in different normal tissues by using WGBS data set from ten adult tissues of one human donor [9] and found that Zone 2 and 4 show lower methylation variation than Zone 1, 3 and 5 (Additional file 1: Fig. S1D). Further, Methylation profiles of buff coat from 15 individuals show same trends (Additional file 1: Fig. S1E). These results indicate that methylations of rDNA IGS region is more stable across tissue types and individuals, which means differences occurred in IGS can be easier to be detected

Based on the findings in normal tissues, we were curious about whether IGS's hypo-methylation is a common feature for different cancer types. The majority of IGS (Zone 2 and 4) shows hypomethylation in at least 4 types of cancer compared with corresponding normal tissues (Fig. 2B-E). In contrast, hyper-methylation in Zone 1, 3 and 5 is not consistent in different cancer types (Fig. 2B-E).

In summary, Zone 2 and 4 which locate in IGS show low methylation variation in normal tissues and significant differential methylation levels in 4 types of cancer. Because plasma cfDNA is a mix of cfDNA that is released from multiple tissues of human body, low methylation variation of IGS may mean stable signals in healthy plasma, which is benefit for detecting cancer. In following sections, we would step forward to investigate the methylation in plasma cfDNA and examined the possibility of rDNA's IGS being used as cancer detection markers. 


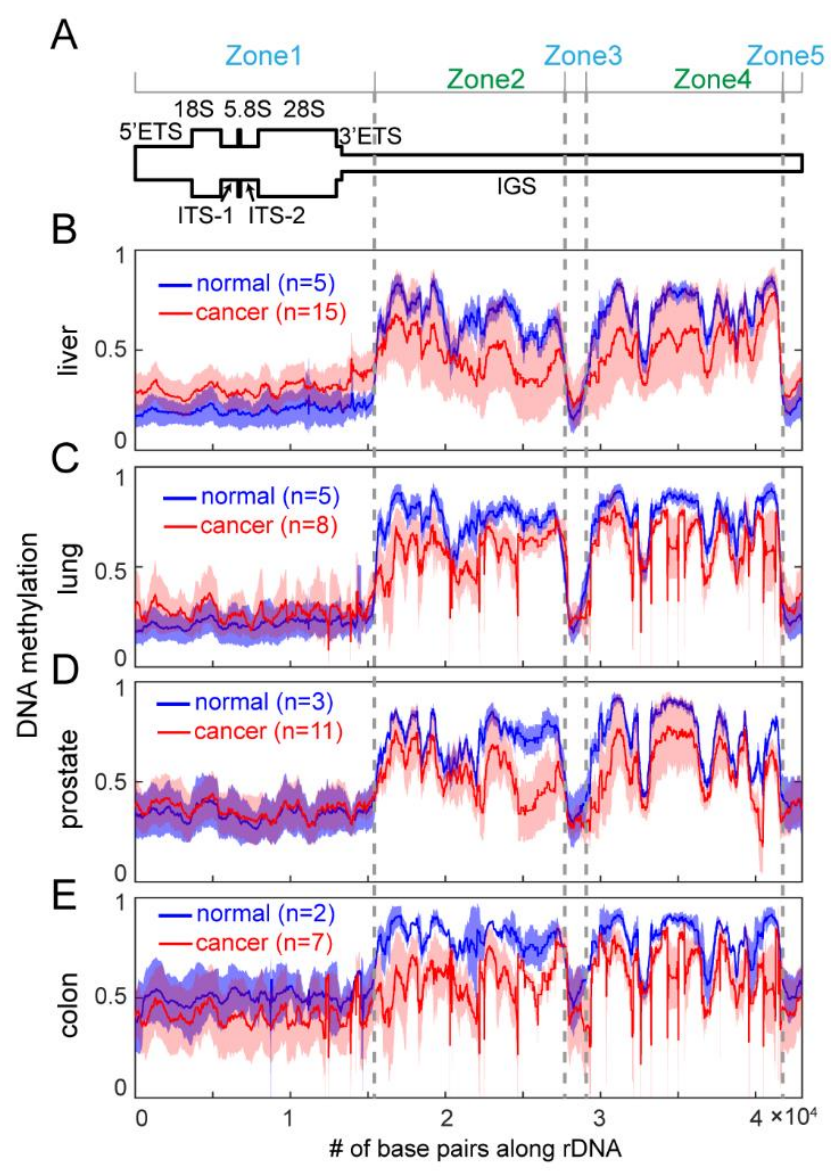

Figure 2 methylation levels in rDNA for 4 cancer types

(A) A schematic representation of human rDNA repeat unit. (B-E) Plots of methylation levels along $r D N A$ reference sequences for four different cancer types and corresponding normal tissues. The red and blue lines are the mean methylation levels of tumors and normal tissues. Scopes of plots show the methylation intervals of \pm 1 standard derivation.

\section{IGS of rDNA showed hypo-methylation in plasma samples from cancer patients}

In liquid biopsy, cfDNA from plasma is one of the most representative analysts which has been widely studied. Plasma cfDNA of healthy subjects comes from various tissues but predominately from blood cells $[45,46]$. Robust and accurate detection of cancer in plasma highly depends on the mix ratio of cancer signals and the stability of markers.

At first, Methylation of plasma cfDNA from healthy individuals showed that Zone 1, 3 and 5 have relatively low methylation level and high methylation variation, while Zone 2 and 4 have high methylation level and very low variations, which is similar to normal tissues (Fig. 3B-C). Further, the top ranked differential $\mathrm{CpG}$ sites between plasma from healthy subjects and cancer patients are more significant than random shuffling background (Additional file 1: Fig. S2A), which suggests that such differences between healthy subjects and cancer patients are not random noises.

Analysis also showed that the top significant candidate markers are mainly located in Zone 2 and Zone 4 of IGS in four types of cancer (Fig. 3D). Plasma from cancer patients also showed hypomethylation in Zone 2 and Zone 4 (Additional file 1: Fig. S3A), which shows the consistent direction of changes with tumor tissues. Two examples of $\mathrm{CpG}$ sites which locate at Zone 2 and 4 show hypomethylation in cancer plasma samples, while there are no significant differences between HBV 
carriers and healthy subject (Fig. 3E-F).

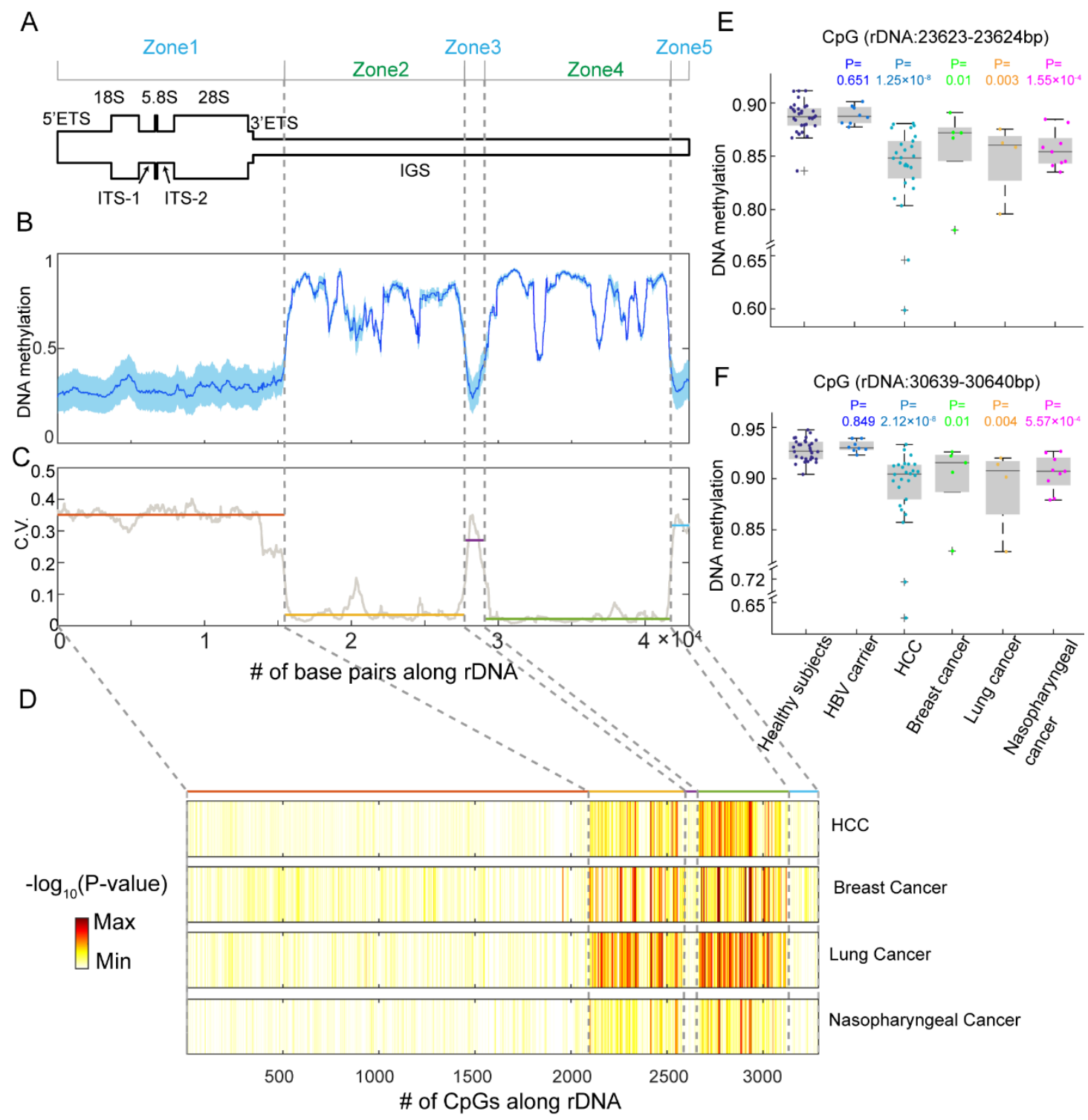

Figure 3 rDNA's methylation of plasma cfDNA

(A) A schematic representation of human rDNA repeat unit. (B) Plots of methylation levels for plasma cfDNA of healthy subjects. The line indicates the mean methylation level of healthy plasma. Scopes of plots show the methylation intervals of \pm 1 standard derivation. (C) Plot of coefficient of variance (C.V.) of healthy plasma in rDNA. For each zone, median of C.V. is drew as horizontal line. (D) Heat maps of significance of differences in distinguishing healthy and cancer patients on single $C p G$ dinucleotides. $C p G$ dinucleotides are ranked by positions on $r D N A$. grey dashed lines show the correspondence between positions and ranked numbers of $C p G$ dinucleotides. (E-F) Boxplots of methylation of two examples of CpG sites at rDNA (23623-23624bp, 30639-30640) in different plasma samples. $P$ values of Wilcoxon test are labeled on the top of samples.

Further, we interrogated differential $\mathrm{CpG}$ dinucleotides throughout comparison between healthy subjects and lung, colon cancer with plasma RRBS data. Though RRBS data prefer genomic regions of high $\mathrm{CpG}$ density, plasma from patients of lung cancer and colon cancer also show hypo- 
methylation at Zone 2 and 4 (Additional file 1: Fig. S3A). And the top ranked significant CpG sites show higher differences than random shuffling (Additional file 1: Fig. S2B). This indicated that hypo-methylation of $\mathrm{CpG}$ sites within IGS is a universal pattern in different cancer types. Methylation levels of CpG sites on IGS can serve as markers for cancer detection in plasma.

\section{rDNA methylation as markers for cancer detection}

In order to evaluate the performances of methylation of rDNA in cancer prediction, we randomly divided the datasets into training and testing sets with a ratio of $2: 1$, and trained classifiers on training dataset and then evaluated the performances on testing dataset. The operation was repeated for 50 times independently to avoid random effects (see Methods). Four classifiers (L1 regularized logistic regression classifier, support vector machine (SVM) classifier, k-nearest neighbor (KNN) classifier and random forest classifier) were used for evaluation.

Since there is only small amount of plasma WGBS samples for lung cancer, breast cancer and nasopharyngeal cancer (see Additional file 2), we performed training and testing only with liver cancer WGBS. For plasma RRBS, we performed the operation on both lung cancer and colon cancer. At first, prediction performance is affected by the number of $\mathrm{CpG}$ sites used as markers. The mean of AUCs could achieve 0.85 with only one $\mathrm{CpG}$ site, and 0.92 with two $\mathrm{CpG}$ sites using liver plasma WGBS (Fig. 4A). The prediction performance becomes stable when the number of CpG sites is 10 . Similar result was observed on plasma RRBS (Additional file 1: Fig. S4A). Therefore, we chose 10 $\mathrm{CpG}$ sites as markers of classifiers in following study. The $\mathrm{CpG}$ sites selected by machine learning mainly located in Zone 2 and 4 of IGS (Additional file 3-5).

Training area under curves (AUCs) are almost 1 and testing AUCs are more than 0.94 in liver cancer with all four classifiers (Additional file 1: Fig. S5A, Fig. 4B). rDNA methylation shows competitive performance in liver cancer detection compared with alpha-fetoprotein (AFP) which was widely used in clinical with AUC of 0.80[47], six-DNA methylation-marker panel with AUC of 0.94[47] and whole genome hypo-methylation method with AUC of 0.93[8]. Similar to liver cancer WGBS, four models training on lung cancer RRBS and colon cancer RRBS showed fairly good performances both in training and testing sets compared with original report of this dataset [9] (Additional file 1. S5B-C, Fig. 4C-D). For example, testing AUCs achieved 0.94 and 0.92 respectively (Fig. 4C-D). Above all, these results suggested that methylation of IGS in rDNA could predict whether a patient has cancer with high accuracy.

Although methylation of rDNA showed high prediction performance in single cancer type, we wondered whether $\mathrm{CpG}$ sites for cancer detection could transfer from one cancer type to others. Classifiers trained with one cancer type predicted effectively for other cancer types within WGBS or RRBS data set (Additional file 1: Fig. S6A-C). Especially in RRBS dataset of lung cancer and colon cancer, models trained with the other cancer type could obtained similar performances as models trained with the same cancer type data (Additional file 1: Fig. S6B-C). And the biomarkers selected using WGBS showed good prediction performance on RRBS data, and vice versa (Additional file 1. S7A-D, 8A-D). However, when we trained models to distinguish lung cancer samples from colon cancer samples with RRBS data, the performance was poor (Additional file 1: Fig. S9A-B). These results suggested that methylation of plasma rDNA might act as potential markers for pan-cancer detection. 
A
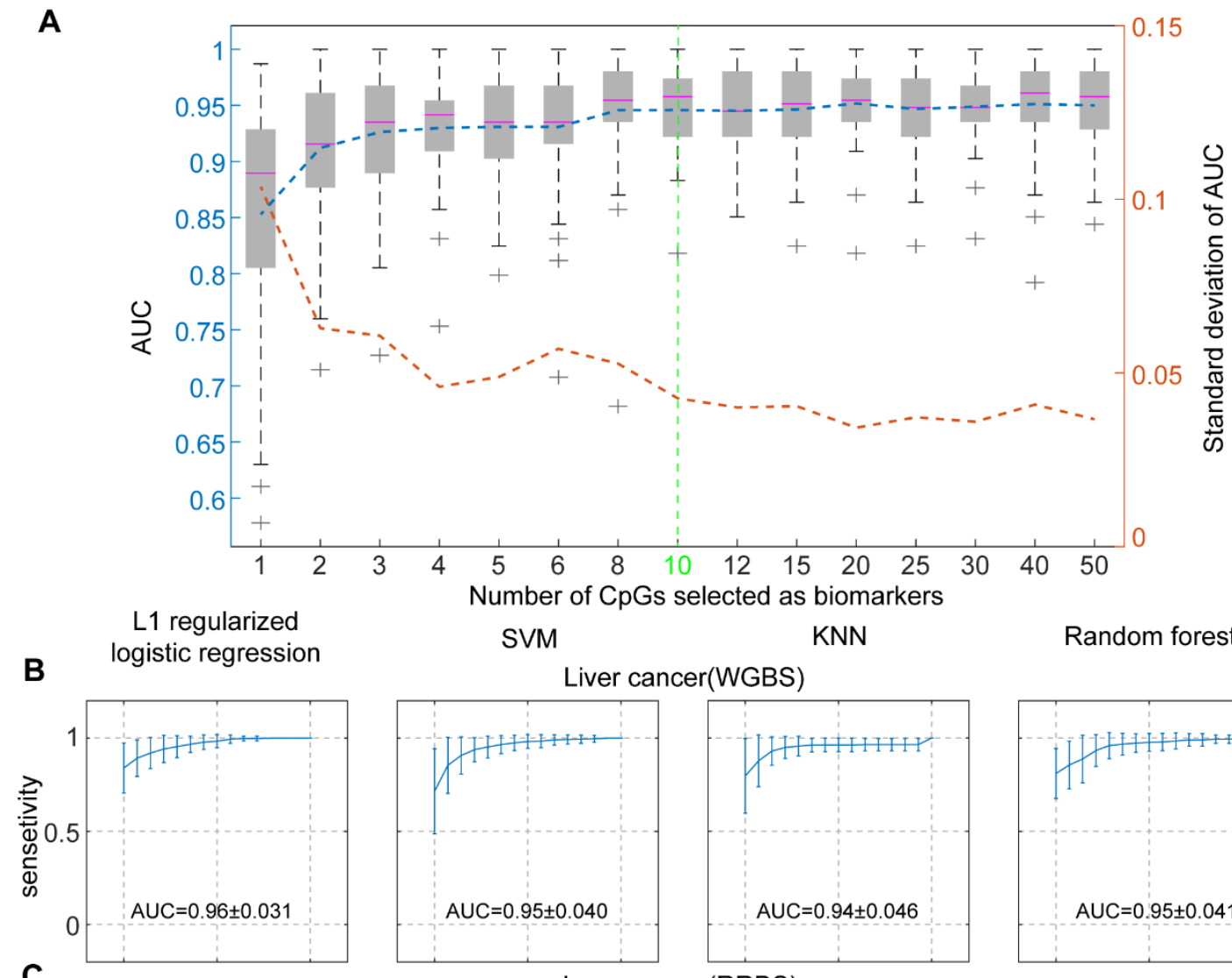

C

SVM

KNN

Random forest

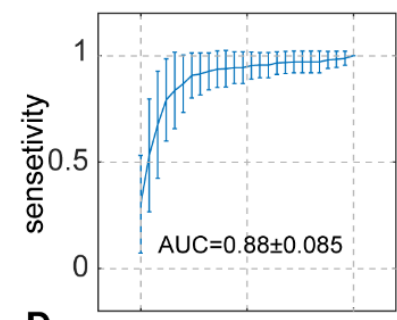

D
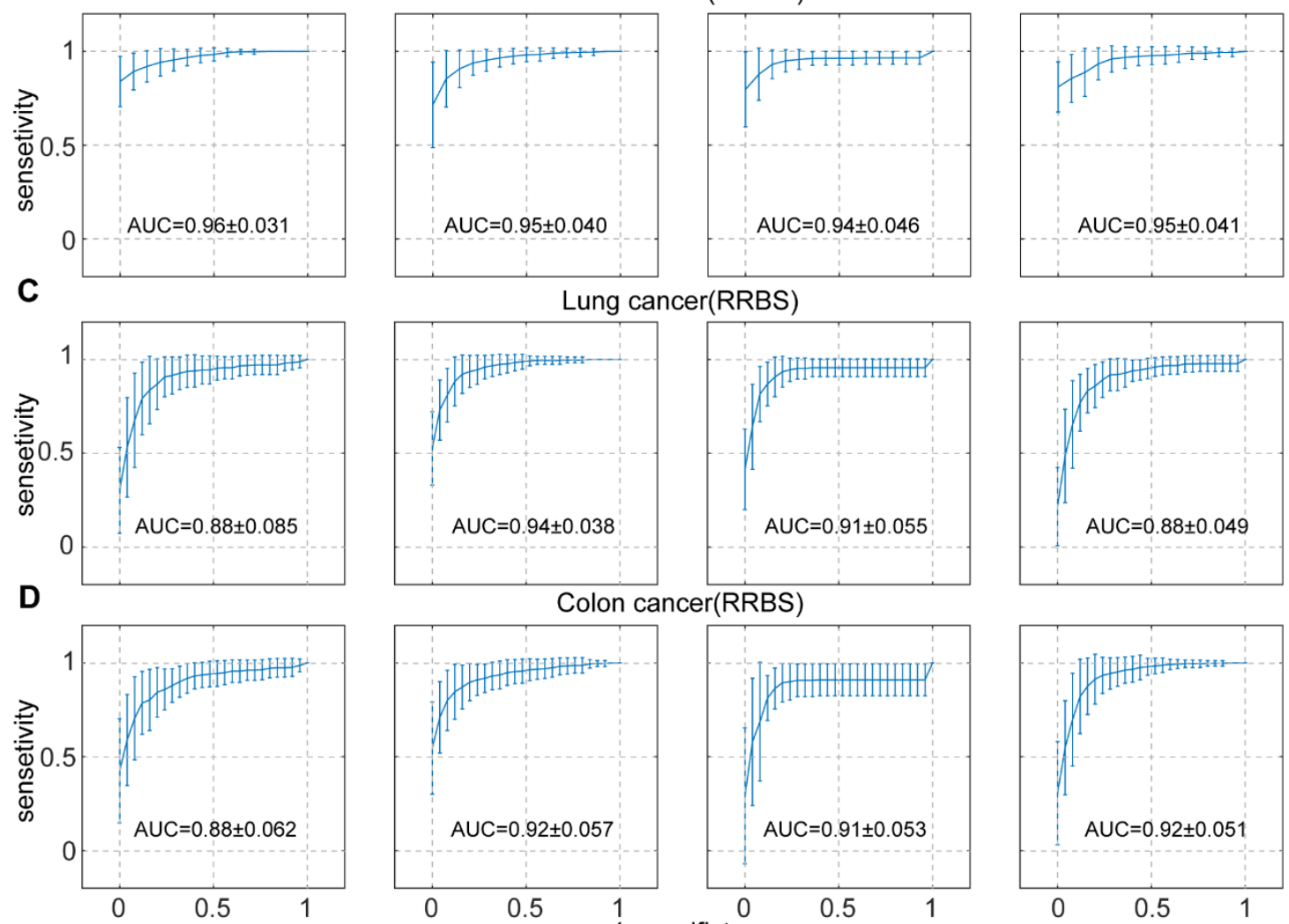

Lung cancer(RRBS)
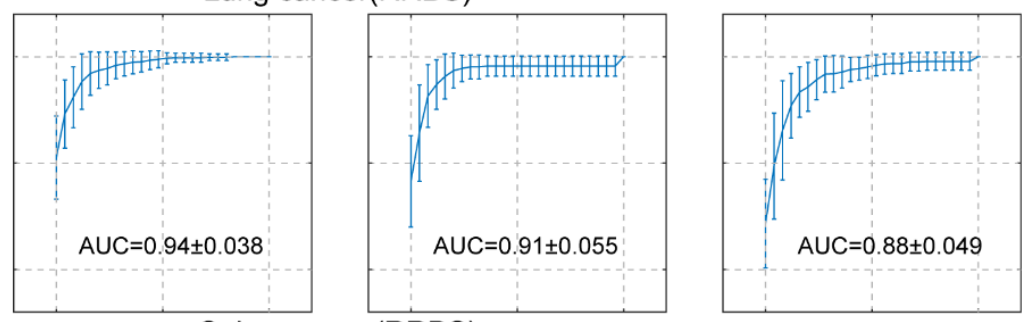

Colon cancer(RRBS)
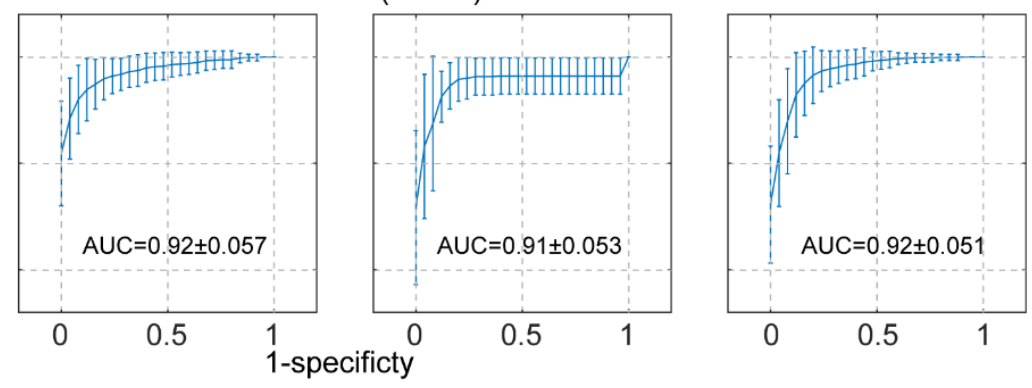

Figure 4 Performances in distinguishing healthy and liver cancer plasma.

(A) Boxplots of AUCs of SVM classifier with different numbers of Cp G sites. Blue dashed line shows mean AUCs, while red dashed line shows AUCs'standard derivation. (B) Receiver operating characteristic (ROC) curves of four classifiers for liver plasma WGBS testing set. Standard derivation bars are labeled with the performance variations of 50 runs. Names of classifiers are labeled on the top of ROC curves. AUCs are labeled on the plots with format of mean \pm 1 standard derivation. (C) ROC curves of four classifiers for lung plasma RRBS testing set. (D) ROC curves of four classifiers for colon plasma RRBS testing set.

Robustness of rDNA methylation as cancer detection markers 
We have mentioned that one great advantage of rDNA for acting as cancer-detection markers is its high copy number. High copy number may enhance the robustness of signal. WGBS covers whole genome and is expensive, so we examined whether methylation of rDNA could keep its prediction performance after down-sampling WGBS sequencing reads. Plasma WGBS of liver cancer was sequenced for one lane (mean 163 million (M) raw reads) of an Illumina HiSeq2000 sequencer per sample, we down-sampled raw sequencing reads to $100 \mathrm{M}, 50 \mathrm{M}, 20 \mathrm{M}$ and $10 \mathrm{M}$, then trained and tested with down-sampled raw sequencing reads (see Methods).

Four classifiers showed slightly decreased performances with down-sampled reads (Fig. 5A). However, even with 10 million raw reads $(0.25 \times$ whole genome coverage), four classifiers could obtain a mean AUC of at least 0.90. L1 regularized logistic regression and random forest classifiers' mean AUC could reach 0.93. It indicated that rDNA methylation acting as cancer detection markers is robust. Robustness of rDNA methylation means that we can reduce the sequencing depth of WGBS to reduce cost of sequencing for following studies or clinical applications.

The number of being selected as markers were counted for each $\mathrm{CpG}$ site in feature selection. With different sequencing depths, $\mathrm{CpG}$ sites which were frequently selected as markers were consistent (Fig. 5B). CpG sites at rDNA: 36996-36997bps, rDNA: 38974-38975bps, rDNA: 37148-37149bps showed high frequencies of being selected in both high and low sequencing depths. CpG sites at rDNA: 37020-37021bps and rDNA: 37013-37014bps showed much higher frequencies in low sequencing depth. $\mathrm{CpG}$ sites being selected in down-sampling dataset mainly locate at Zone 2 and 4 of IGS.

In summary, methylation of IGS in rDNA serves as robust markers for cancer detection in plasma.

A

A L1 regularized logistic regression

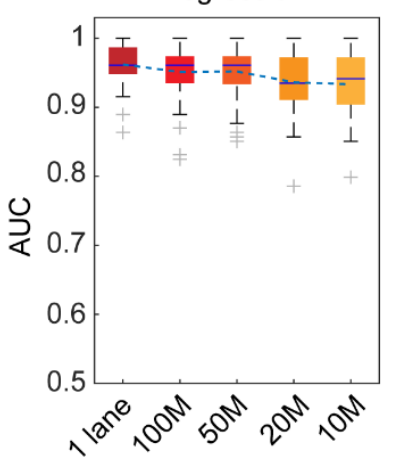

SVM

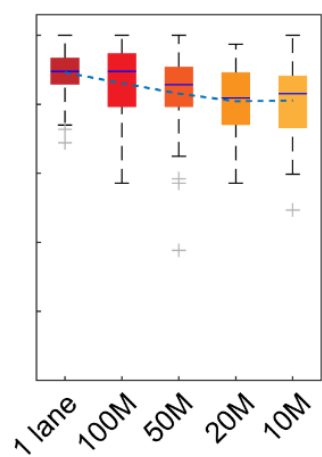

KNN

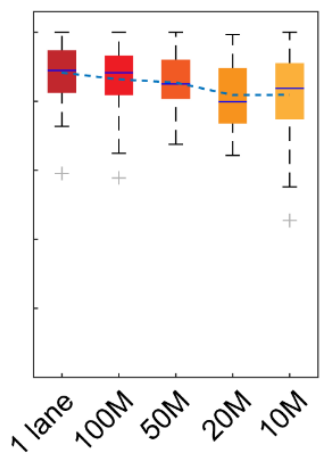

Random forest

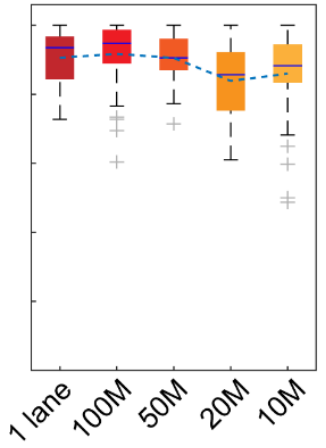

B
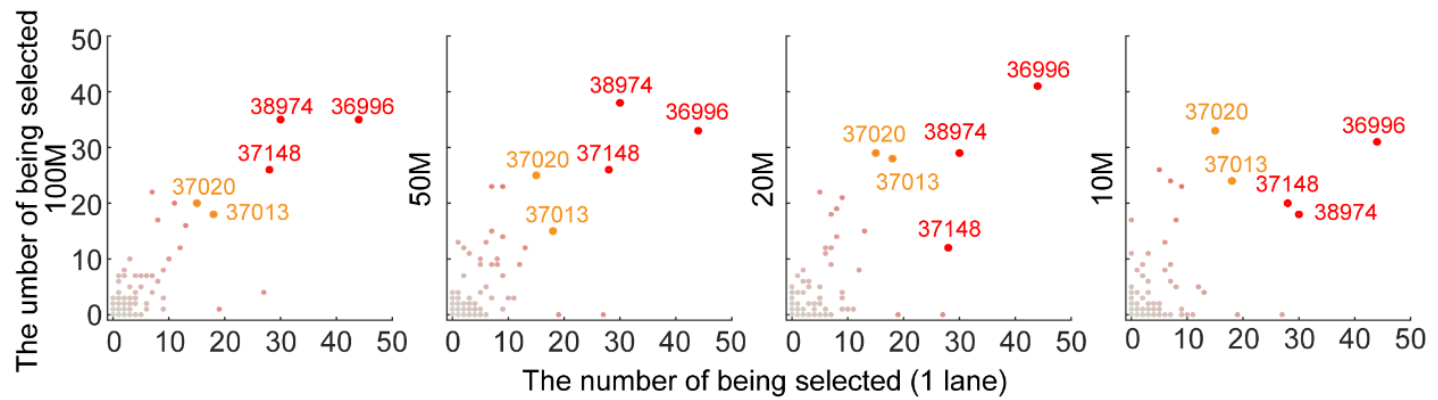

Figure 5 Prediction performances based on down sampling sequencing reads

(A) Boxplots of AUCs in different down sampling depths for four models. Horizontal axis shows the number of raw reads after down sampling. 1 lane is the original of sequencing depth (mean depth: 
163 million). Models are trained and tested with corresponding down-sequencing depths. (B) Scatter plots of the number of being selected as markers for each $C p G$ site in different sequencing depths. Horizontal axis of four scatter plots is the number of being selected for each CpG site with 1 lane sequencing depth. Ordinate is the number of being selected for each CpG site with 100M, 50M, 20M and 10M sequencing depths. Red dots indicate three CpG sites with high selected numbers in both high and low sequencing depths. Orange dots shows two CpG sites with higher selected number in low sequencing depth. The first position of each CpG site in rDNA is labeled around $C p G$ dot.

\section{Methylation of rDNA acting as markers for monitoring cancer progression and treatment}

We also investigated whether methylation of rDNA could be used for monitoring the cancer progression and surgery. There were plasma WGBS of time series from two liver cancer patients, which included plasma WGBS before and after tumor resection. L1-regularized logistic regression classifier trained with liver cancer plasma samples was used to evaluate the effects of surgery. The patient TBR34 died of metastatic disease 8 months after surgery[8], which was consistent with the prediction that TBR34 still had high probability of having cancer after surgery (Fig. 6A). The patient TBR36 was still alive beyond 20 months after surgery[8], which was consistent with the fact that the predicted probability of having caner decreased obviously 3 days after surgery (Fig. 6A).

Further, performance of down-sampling sequence reads was checked and the prediction results in different down-sampling sequencing depths were similar to that of no down-sampling data (Fig. 6BE). The results mean that we can evaluate the surgery effects with low sequencing depth and take measures ahead of cancer progression. Taken together, methylation of rDNA can also be used as robust markers for monitoring cancer progression and treatment.

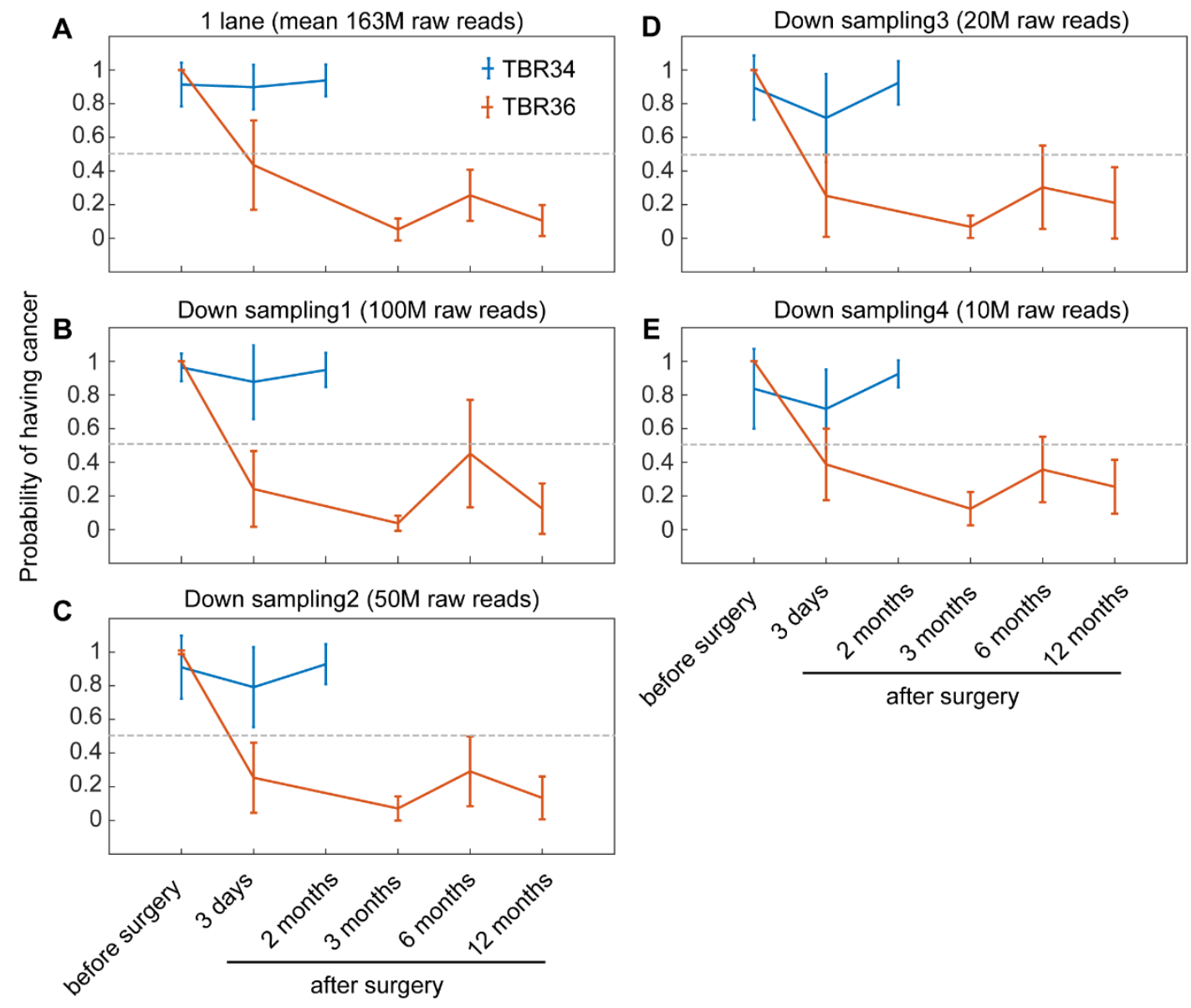


bioRxiv preprint doi: https://doi.org/10.1101/651497; this version posted May 29,2019 . The copyright holder for this preprint (which was not certified by peer review) is the author/funder, who has granted bioRxiv a license to display the preprint in perpetuity. It is made available under aCC-BY-NC-ND 4.0 International license.

\section{Figure 6 Monitoring cancer progression for two patients}

(A) Bar boxplots of predicted probabilities of having cancer for patient TBR34 and TBR36. Horizontal axis shows the time lines of tumor resection. Horizontal grey line shows the threshold of 0.5. (B-E) Bar boxplots of predicted probabilities of having cancer for two patients based on different down sampling depths. The predicted models are trained and tested in corresponding down-sequencing depths.

\section{Discussion}

In this study, we used WGBS and RRBS data to investigate the methylation patterns of rDNA at each single $\mathrm{CpG}$ site for multiple cancer types and identified the aberrant methylation patterns of cancer. We found that $\mathrm{CpG}$ sites within IGS are promising markers for cancer detection. These $\mathrm{CpG}$ sites have high methylation level and low variation both in normal tissues and plasma, while have decreased methylation levels in four cancer types. We evaluated the performance of these markers with plasma bisulfite sequencing samples from healthy subjects and cancer patients. Further, we found these markers could also be used for monitoring cancer progression and treatment.

In this study, we showed that hypo-methylation of reduced IGS is a universal feature in 4 cancer types, which was rarely reported before. In previous studies, researchers kept focusing on methylation changes of promoter or transcription body of rDNA. In breast cancer, ovarian cancer and endometrial carcinoma, hyper-methylation of transcribed domain of rDNA were reported as an important feature and its methylation level was correlated with patients' survival rates[37-39]. However, this feature can hardly be used as markers in plasma biopsy because methylation of these regions has high variation in both plasma samples of healthy subjects and normal tissues. In contrast, CpG sites in IGS will be attractive biomarkers for both solid biopsy and liquid biopsy.

Using rDNA methylation as markers for cancer detection has one obvious advantage - robustness. Studying the methylation pattern of $\mathrm{CpG}$ sites in non-repeated genome regions required very high coverage to get stable signals. In this study, down-sampling experiments showed that sequencing depth of 10 million raw reads $(0.25 \times$ whole genome coverage) could also obtain competitive prediction performance due to high copy number, which provides us a robust biomarker. In addition to non-targeted bisulfite sequencing, designing probes or primers that target the differential $\mathrm{CpG}$ sites in rDNA will be an alternative way.

However, there were some limitations in this study, which need to be optimized for better outputs with the developments of technologies and databases. First, sequencing reads were mapped to only one reference rDNA sequence and signals were aggregated for all copies of rDNA in this study. However, it was reported that rDNA sequence has a certain rate of mutations, especially in IGS[13]. Recently, two studies has explored the sequence variation in $\operatorname{rDNA}[48,49]$. Researchers studied chromosome 21 of one individual and identified 101 and 235 variant positions in transcribed regions and IGS respectively[49]. By analyzing whole genome sequencing data from 1000 Genome Project, researchers also identified intra- and inter-individual nucleotide variations in transcribed regions and found variant ribosomal RNA alleles might be correlated with physiology and diseases[48]. With the development of human genetic variants about rDNA, sequencing reads can be mapped to multiple rDNA alleles, which may assist us to investigate the methylation patterns of rDNA and detect cancer more accurately.

In addition, fragments with low methylation levels are more easy to be damaged during bisulfite treatment in library construction[50]. It means that hypo-methylation of cancer tissues may be more 
hard to be detected due to bisulfite conversion. If there are alternative technologies which retain the hypo-methylation fragments, it may improve the power of rDNA's methylation in cancer detection. For example, third generation sequencing can detect DNA methylation without any processing on DNA samples[51].

Finally, methylation of rDNA is a potential marker for pan-cancer detection and can be used for screening potential cancer patients. However, it may need to combine with other cancer typespecific markers to achieve cancer type-specific predictions. It has been suggested that combining multiple parameters or analysts enhances the performance of liquid biopsy[1,2]. Therefore, methylation of rDNA can serve as perfect partner markers for cancer managements.

\section{Conclusion}

In this study, we provided high-resolution map of DNA methylation at each single $\mathrm{CpG}$ site for cancer with bisulfite sequencing data. We found that $\mathrm{CpG}$ sites in IGS of rDNA has high methylation level and low variation in both normal tissues and plasma and showed hypo-methylation in 4 cancer types. The study supported that methylation of IGS in rDNA provides a competitive and robust marker for detecting cancer and monitoring cancer progression and treatment.

\section{Methods}

\section{Data collection}

We collected WGBS or RRBS data of tumors, normal tissues and plasma cfDNA from published dataset[ $8,9,52,53]$. In total, we obtained 15 types of normal tissues and 4 types of cancer (see Additional file 2). Cancer types were selected based on that the number of samples from one cancer type is more than 5. In this study, we analyzed 41 samples of normal tissues and 45 samples of tumors and investigated the methylation patterns in rDNA. We also collected WGBS and RRBS data of plasma cfDNA from healthy subjects and patients with 5 different cancer types (see Additional file 2). There are totally 224 plasma cfDNA samples. These data were downloaded from Gene Expression Omnibus (GEO) under accession number GSE52272, GSE70091, GSE79279, GSE104789 and European Genome-Phenome Archive (EGA) under accession number EGAS00001000566.

\section{Analyses of WGBS and RRBS data}

Downloaded data were mapped to human ribosomal DNA repeat unit reference sequences (Genebank: U13369.1, https://www.ncbi.nlm.nih.gov/nuccore/U13369.1) with software BSSeeker2[54]. We used 'local alignment' mode to map these data. Mapped bam files were used to call methylations at single $\mathrm{CpG}$ dinucleotides without removing duplications because there are extremely high coverages in rDNA. We only considered the methylation patterns of $\mathrm{CpG}$ dinucleotides as there were low methylation levels at non-CpG sites in human genome. We removed mapped reads which had alignment lengths less than 40 bps. Too small alignment length caused higher false positive hitting. Then, the numbers of methylation and un-methylation reads were counted at each single $\mathrm{CpG}$ sites. Finally, the methylation levels of each $\mathrm{CpG}$ sites were calculated unless the mapped reads number in each sample was less 20.

\section{Dividing rDNA into different zones}

Different regions in rDNA showed different methylation patterns. In order to describe the 
methylation patterns of rDNA, we divided rDNA into 5 different non-overlapped zones according to methylation of $\mathrm{CpG}$ sites. First, we calculated the methylation levels and correlation matrix of $\mathrm{CpG}$ sites in normal tissues and tumors. Next, we identified boundaries of $\mathrm{CpG}$ sites of different zones roughly. We also calculated PC1 of correlation matrix by performing PCA on correlation matrix. We identified the exact $\mathrm{CpG}$ site boundaries by shifting rough boundaries several $\mathrm{CpG}$ sites to achieve the largest differences of median PC1 between adjacent zones. Finally, boundary coordinates were determined according to $\mathrm{CpG}$ boundaries.

\section{Training classifiers and predicting}

For liver cancer, we randomly divided healthy subjects, HBV carrier and liver cancer patients into training and testing sets with a ratio of 2:1. In training set, we screened the markers and trained classifiers; in testing set, we validated the chosen markers and models trained with training set.

In training set, we drew ROC curve and calculate the AUC for each $\mathrm{CpG}$ site in distinguishing cancer patients and non-cancer subjects. The higher the AUC was, the better distinguishing effect was. We sorted the $\mathrm{CpG}$ sites according to the AUC scores and chose the top 200 ranked markers. Then we used SVM classifier to select a certain number of features (CpG sites) from top 200 ranked markers with training dataset. In order to make sure that selected differential markers in plasma were due to the differences of liver tumor tissues and normal tissues, we checked the methylation pattern of markers in liver tumor tissues and buff coat. We tried several classification methods to combine the selected markers for predicting whether a person is a cancer patient. These classification methods included L1-regularied logistic regression, supported vector machine, knearest neighbor and random forest.

In testing set, we validated the screened markers and trained models by calculating AUCs. In order to avoid random noises, we randomly divided the datasets for 50 times independently and computed the mean AUCs in 50 independent training-testing cases.

For lung cancer, breast cancer and nasopharyngeal cancer which had small number of WGBS samples, we didn't perform the training and testing operations. For lung cancer and colon cancer which had enough numbers of RRBS samples, the training and testing steps were the similar as liver cancer.

\section{Down-sampling sequencing reads}

In order to test the robustness of markers in rDNA, we down-sampled WGBS data of healthy, HBV carrier and liver cancer plasma samples into different sequencing depths. Each sample of plasma WGBS used one lane of an Illumina Hiseq 2000 sequencer and obtained an average of 163 million raw reads. We down-sampled raw sequencing reads into 100 million, 50 million, 20 million and 10 million subsets. Based on the down-sampled raw reads, we performed the same training and testing steps to study the robustness of rDNA markers. As RRBS data had been enriched signals coming from CpG-rich regions through adjusted biological technology, down-sampling was not applied to RRBS data.

\section{Additional files}

Additional file 1: Supplementary Materials

Additional file 2: Data collection information

Additional file 3: $\mathrm{CpG}$ sites selected for liver cancer detection (WGBS) 
Additional file 4: CpG sites selected for lung cancer detection (RRBS)

Additional file 5: CpG sites selected or colon cancer detection (RRBS)

\begin{abstract}
Abbreviations
AFP: alpha-fetoprotein; AUC: area under curves; bp: base pair; cfDNA: cell free DNA; CpG: cytosine-phosphate-guanine; ctDNA: circulating tumor DNA; C.V.: coefficient of variance; ETS: external transcribed spacer; IGS: intergenic spacer; ITS: internal transcribed spacer; KNN: k-nearest neighbor; M: million; rDNA: ribosomal DNA; ROC: Receiver operating characteristic; RRBS: reduced representation bisulfite sequencing; SVM: support vector machine (SVM) classifier; WGBS: Whole genome bisulfite sequencing.
\end{abstract}

\title{
Acknowledge
}

The authors greatly acknowledge Dr. Yuk Ming Dennis Lo and his research group in the Chinese University of Hong Kong for providing the cfDNA WGBS data [8].

\section{Funding}

This work was supported by National Science Foundation of China Grants (No. 61773230, 61721003).

\section{Availability of data and materials}

The original datasets analyzed in the current study were reported previously $[8,9,52,53]$ and can be downloaded from Gene Expression Omnibus (GEO) under accession number GSE52272, GSE70091, GSE79279, GSE104789 and European Genome-Phenome Archive (EGA) under accession number EGAS00001000566. The list of accessions and corresponding cancer types in the current study were provided in Additional file 2. The $\mathrm{CpG}$ sites which were selected as biomarkers were provided in Additional file 3-5.

\section{Authors' contributions}

$\mathrm{XZ}$ and XW conceived the study and designed the methodological framework. XZ collected, processed the WGBS and RRBS data and performed followed analyses. XZ wrote the manuscript with input from XW, HF, WZ, BZ and YL. All authors read and approved the final manuscript.

\section{Ethics approval and consent to participate}

Not applicable

\section{Consent for publication}

Not applicable

\section{Competing interests}

Tsinghua university has a patent pending for some CpG sites in rDNA. The patent, however, does not restrict the research use.

\section{Author details}

Department of Automation, Center for Synthetic and Systems Biology, Tsinghua University; Ministry of Education Key Laboratory of Bioinformatics; Bioinformatics Division, BNRIST, 
bioRxiv preprint doi: https://doi org/10.1101/651497; this version posted May 29 2019. The copyright holder for this preprint (which was not certified by peer review) is the author/funder, who has granted bioRxiv a license to display the preprint in perpetuity. It is made available under aCC-BY-NC-ND 4.0 International license.

Beijing 100084, China

\section{Reference}

1. Wan JCM, Massie C, Garcia-Corbacho J, Mouliere F, Brenton JD, Caldas C, Pacey S, Baird R, Rosenfeld N: Liquid biopsies come of age: towards implementation of circulating tumour DNA. Nat Rev Cancer 2017, 17(4):223-238.

2. Heitzer E, Haque IS, Roberts CES, Speicher MR: Current and future perspectives of liquid biopsies in genomics-driven oncology. Nat Rev Genet 2019, 20(2):71-88.

3. Bardelli A, Pantel K: Liquid Biopsies, What We Do Not Know (Yet). Cancer Cell 2017, 31(2):172-179.

4. Warton K, Mahon KL, Samimi G: Methylated circulating tumor DNA in blood: power in cancer prognosis and response. Endocr Relat Cancer 2016, 23(3):R157-171.

5. Berdasco M, Esteller M: Clinical epigenetics: seizing opportunities for translation. Nat Rev Genet 2019, 20(2):109-127.

6. Pan YB, Liu GH, Zhou FL, Su BJ, Li YR: DNA methylation profiles in cancer diagnosis and therapeutics. Clin Exp Med 2018, 18(1):1-14.

7. Fan S, Chi W: Methods for genome-wide DNA methylation analysis in human cancer. Brief Funct Genomics 2016, 15(6):432-442.

8. Chan KC, Jiang P, Chan CW, Sun K, Wong J, Hui EP, Chan SL, Chan WC, Hui DS, Ng SS et al: Noninvasive detection of cancer-associated genome-wide hypomethylation and copy number aberrations by plasma DNA bisulfite sequencing. Proc Natl Acad Sci U $S$ A 2013, 110(47):18761-18768.

9. Guo S, Diep D, Plongthongkum N, Fung HL, Zhang K, Zhang K: Identification of methylation haplotype blocks aids in deconvolution of heterogeneous tissue samples and tumor tissue-of-origin mapping from plasma DNA. Nat Genet 2017, 49(4):635-642.

10. Leygo C, Williams M, Jin HC, Chan MWY, Chu WK, Grusch M, Cheng YY: DNA Methylation as a Noninvasive Epigenetic Biomarker for the Detection of Cancer. Dis Markers 2017.

11. Tang QQ, Cheng J, Cao X, Surowy H, Burwinkel B: Blood-based DNA methylation as biomarker for breast cancer: a systematic review. Clinical Epigenetics 2016, 8.

12. Mallik S, Zhao Z: Towards integrated oncogenic marker recognition through mutual information-based statistically significant feature extraction: an association rule mining based study on cancer expression and methylation profiles. Quant Bio/2017, 5(4):302-327.

13. Gonzalez IL, Sylvester JE: Complete Sequence of the 43-Kb Human Ribosomal DNA Repeat - Analysis of the Intergenic Spacer. Genomics 1995, 27(2):320-328.

14. Agrawal S, Ganley ARD: The conservation landscape of the human ribosomal RNA gene repeats. Plos One 2018, 13(12):e0207531.

15. Tsekrekou M, Stratigi K, Chatzinikolaou G: The Nucleolus: In Genome Maintenance and Repair. Int J Mol Sci 2017, 18(7).

16. van Sluis M, McStay B: Nucleolar reorganization in response to rDNA damage. Curr Opin Cell Biol 2017, 46:81-86.

17. Gibbons JG, Branco AT, Godinho SA, Yu S, Lemos B: Concerted copy number variation balances ribosomal DNA dosage in human and mouse genomes. Proc Natl Acad SCI 
bioRxiv preprint doi: https://doi org/10.1101/651497; this version posted May 29 2019. The copyright holder for this preprint (which was not certified by peer review) is the author/funder, who has granted bioRxiv a license to display the preprint in perpetuity. It is made available under aCC-BY-NC-ND 4.0 International license.

U S A 2015, 112(8):2485-2490.

18. Gibbons JG, Branco AT, Yu S, Lemos B: Ribosomal DNA copy number is coupled with gene expression variation and mitochondrial abundance in humans. Nat Commun 2014, 5:4850.

19. Wang M, Lemos B: Ribosomal DNA copy number amplification and loss in human cancers is linked to tumor genetic context, nucleolus activity, and proliferation. Plos Genet 2017, 13(9).

20. Xu B, Li H, Perry JM, Singh VP, Unruh J, Yu Z, Zakari M, McDowell W, Li L, Gerton JL: Ribosomal DNA copy number loss and sequence variation in cancer. Plos Genet 2017, 13(6):e1006771.

21. Udugama M, Sanij E, Voon HPJ, Son J, Hii L, Henson JD, Chan FL, Chang FTM, Liu Y, Pearson RB et al: Ribosomal DNA copy loss and repeat instability in ATRX-mutated cancers. Proc Natl Acad Sci U S A 2018, 115(18):4737-4742.

22. Hosgood HD, Hu W, Rothman N, Klugman M, Weinstein SJ, Virtamo J, Albanes D, Cawthon R, Lan Q: Variation in Ribosomal DNA copy number is associated with lung cancer risk in a prospective cohort study. Carcinogenesis 2019.

23. Raval A, Sridhar KJ, Patel S, Turnbull BB, Greenberg PL, Mitchell BS: Reduced rRNA expression and increased rDNA promoter methylation in CD34+ cells of patients with myelodysplastic syndromes. Blood 2012, 120(24):4812-4818.

24. Uemura M, Zheng Q, Koh CM, Nelson WG, Yegnasubramanian S, De Marzo AM: Overexpression of ribosomal RNA in prostate cancer is common but not linked to rDNA promoter hypomethylation. Oncogene 2012, 31(10):1254-1263.

25. Karahan G, Sayar N, Gozum G, Bozkurt B, Konu O, Yulug IG: Relative expression of rRNA transcripts and 45S rDNA promoter methylation status are dysregulated in tumors in comparison with matched-normal tissues in breast cancer. Oncol Rep 2015, 33(6):3131-3145

26. Zhou H, Wang YP, Lv QY, Zhang J, Wang Q, Gao F, Hou HL, Zhang H, Zhang W, Li LJ: Overexpression of Ribosomal RNA in the Development of Human Cervical Cancer Is Associated with rDNA Promoter Hypomethylation. Plos One 2016, 11(10).

27. Tsoi H, Lam KC, Dong Y, Zhang X, Lee CK, Zhang J, Ng SC, Ng SSM, Zheng S, Chen Y et al: Pre-45s rRNA promotes colon cancer and is associated with poor survival of CRC patients. Oncogene 2017, 36(44):6109-6118.

28. Lawrence RJ, Earley K, Pontes O, Silva M, Chen ZJ, Neves N, Viegas W, Pikaard CS: A concerted DNA methylation/histone methylation switch regulates rRNA gene dosage control and nucleolar dominance. Molecular Ce//2004, 13(4):599-609.

29. McStay B, Grummt I: The epigenetics of rRNA genes: from molecular to chromosome biology. Annu Rev Cell Dev Bio/2008, 24:131-157.

30. Srivastava R, Srivastava R, Ahn SH: The Epigenetic Pathways to Ribosomal DNA Silencing. Microbiol Mol Biol Rev 2016, 80(3):545-563.

31. Gagnon-Kugler T, Langlois F, Stefanovsky V, Lessard F, Moss T: Loss of human ribosomal gene CpG methylation enhances cryptic RNA polymerase II transcription and disrupts ribosomal RNA processing. Mol Ce//2009, 35(4):414-425.

32. Wang $M$, Lemos B: Ribosomal DNA harbors an evolutionarily conserved clock of biological aging. Genome Res 2019, 29(3):325-333. 
33. D'Aquila P, Montesanto A, Mandala M, Garasto S, Mari V, Corsonello A, Bellizzi D, Passarino G: Methylation of the ribosomal RNA gene promoter is associated with aging and age-related decline. Aging Ce//2017, 16(5):966-975

34. Brock GJR, Bird A: Mosaic methylation of the repeat unit of the human ribosomal RNA genes. Human Molecular Genetics 1997, 6(3):451-456.

35. Shiraishi M, Sekiguchi A, Chuu YH, Sekiya T: Alteration of mosaic methylation of the repeat unit of the human ribosomal RNA genes in lung cancer. Biol Chem 1999, 380(1):81-84.

36. Bacalini MG, Pacilli A, Giuliani C, Penzo M, Trere D, Pirazzini C, Salvioli S, Franceschi C, Montanaro L, Garagnani P: The nucleolar size is associated to the methylation status of ribosomal DNA in breast carcinomas. Bmc Cancer 2014, 14.

37. Yen PS, Rodriguez FJ, Laux DE, Perry MR, Standiford SB, Huang TM: Hypermethylation of ribosomal DNA in human breast carcinoma. Brit J Cancer 2000, 82(3):514-517.

38. Chan MW, Wei SH, Wen P, Wang Z, Matei DE, Liu JC, Liyanarachchi S, Brown R, Nephew KP, Yan PS et al: Hypermethylation of $18 \mathrm{~S}$ and $28 \mathrm{~S}$ ribosomal DNAs predicts progression-free survival in patients with ovarian cancer. Clin Cancer Res 2005, 11(20):7376-7383.

39. Powell MA, Mutch DG, Rader JS, Herzog TJ, Huang TH, Goodfellow PJ: Ribosomal DNA methylation in patients with endometrial carcinoma: an independent prognostic marker. Cancer 2002, 94(11):2941-2952.

40. Toyota M, Ho C, Ahuja N, Jair KW, Li Q, Ohe-Toyota M, Baylin SB, Issa JP: Identification of differentially methylated sequences in colorectal cancer by methylated $\mathrm{CpG}$ island amplification. Cancer Res 1999, 59(10):2307-2312.

41. Yu F, Shen XY, Fan L, Yu ZC: Analysis of histone modifications at human ribosomal DNA in liver cancer cell. Sci Rep-Uk 2015, 5.

42. Zentner GE, Saiakhova A, Manaenkov P, Adams MD, Scacheri PC: Integrative genomic analysis of human ribosomal DNA. Nucleic Acids Res 2011, 39(12):4949-4960.

43. Li E, Zhang Y: DNA methylation in mammals. Cold Spring Harb Perspect Biol 2014, 6(5):a019133.

44. Smith ZD, Meissner A: DNA methylation: roles in mammalian development. Nature Reviews Genetics 2013, 14(3):204-220.

45. Sun K, Jiang P, Chan KC, Wong J, Cheng YK, Liang RH, Chan WK, Ma ES, Chan SL, Cheng $\mathrm{SH}$ et al: Plasma DNA tissue mapping by genome-wide methylation sequencing for noninvasive prenatal, cancer, and transplantation assessments. Proc Natl Acad Sci U $S$ A 2015, 112(40):E5503-5512.

46. Moss J, Magenheim J, Neiman D, Zemmour H, Loyfer N, Korach A, Samet Y, Maoz M, Druid $\mathrm{H}$, Arner $\mathrm{P}$ et al: Comprehensive human cell-type methylation atlas reveals origins of circulating cell-free DNA in health and disease. Nat Commun 2018, 9(1):5068.

47. Kisiel JB, Dukek BA, R VSRK, Ghoz HM, Yab TC, Berger CK, Taylor WR, Foote PH, Giama $\mathrm{NH}$, Onyirioha $\mathrm{K}$ et al: Hepatocellular Carcinoma Detection by Plasma Methylated DNA: Discovery, Phase I Pilot, and Phase II Clinical Validation. Hepatology 2019, 69(3):1180-1192.

48. Parks MM, Kurylo CM, Dass RA, Bojmar L, Lyden D, Vincent CT, Blanchard SC: Variant 
ribosomal RNA alleles are conserved and exhibit tissue-specific expression. Sci AdV 2018, 4(2):eaao0665.

49. Kim JH, Dilthey AT, Nagaraja R, Lee HS, Koren S, Dudekula D, Wood lii WH, Piao Y, Ogurtsov AY, Utani $\mathrm{K}$ et al: Variation in human chromosome 21 ribosomal RNA genes characterized by TAR cloning and long-read sequencing. Nucleic Acids Res 2018, 46(13):6712-6725.

50. Olova N, Krueger F, Andrews S, Oxley D, Berrens RV, Branco MR, Reik W: Comparison of whole-genome bisulfite sequencing library preparation strategies identifies sources of biases affecting DNA methylation data. Genome Bio/2018, 19(1):33.

51. McCarthy $A$ : Third generation DNA sequencing: pacific biosciences' single molecule real time technology. Chem Bio/2010, 17(7):675-676.

52. Li X, Liu Y, Salz T, Hansen KD, Feinberg A: Whole-genome analysis of the methylome and hydroxymethylome in normal and malignant lung and liver. Genome Res 2016, 26(12):1730-1741.

53. Heyn H, Vidal E, Ferreira HJ, Vizoso M, Sayols S, Gomez A, Moran S, Boque-Sastre R, Guil $\mathrm{S}$, Martinez-Cardus A et al: Epigenomic analysis detects aberrant super-enhancer DNA methylation in human cancer. Genome Bio/2016, 17:11.

54. Guo W, Fiziev P, Yan W, Cokus S, Sun X, Zhang MQ, Chen PY, Pellegrini M: BS-Seeker2: a versatile aligning pipeline for bisulfite sequencing data. BMC Genomics 2013, 14:774. 


\section{Additional file 1: Supplementary Materials}

\section{Ribosomal DNA methylation as stable biomarkers for detection of}

\section{cancer in plasma}

Xianglin Zhang, Huan Fang, Wei Zhang, Bixi Zhong, Yanda Li, Xiaowo Wang* Department of Automation, Center for Synthetic and Systems Biology, Tsinghua University; Ministry of Education Key Laboratory of Bioinformatics; Bioinformatics Division, BNRIST,

Beijing 100084, China

*Corresponding author: xwwang@tsinghua.edu.cn 
A

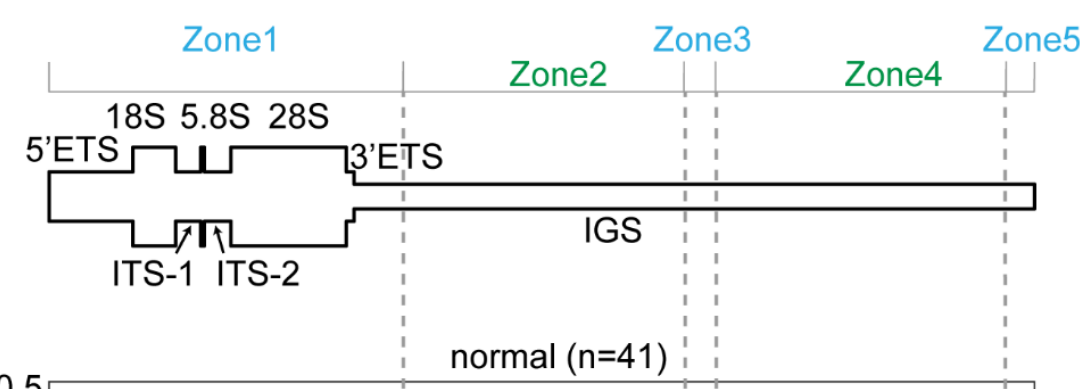

B

0.5
0.4

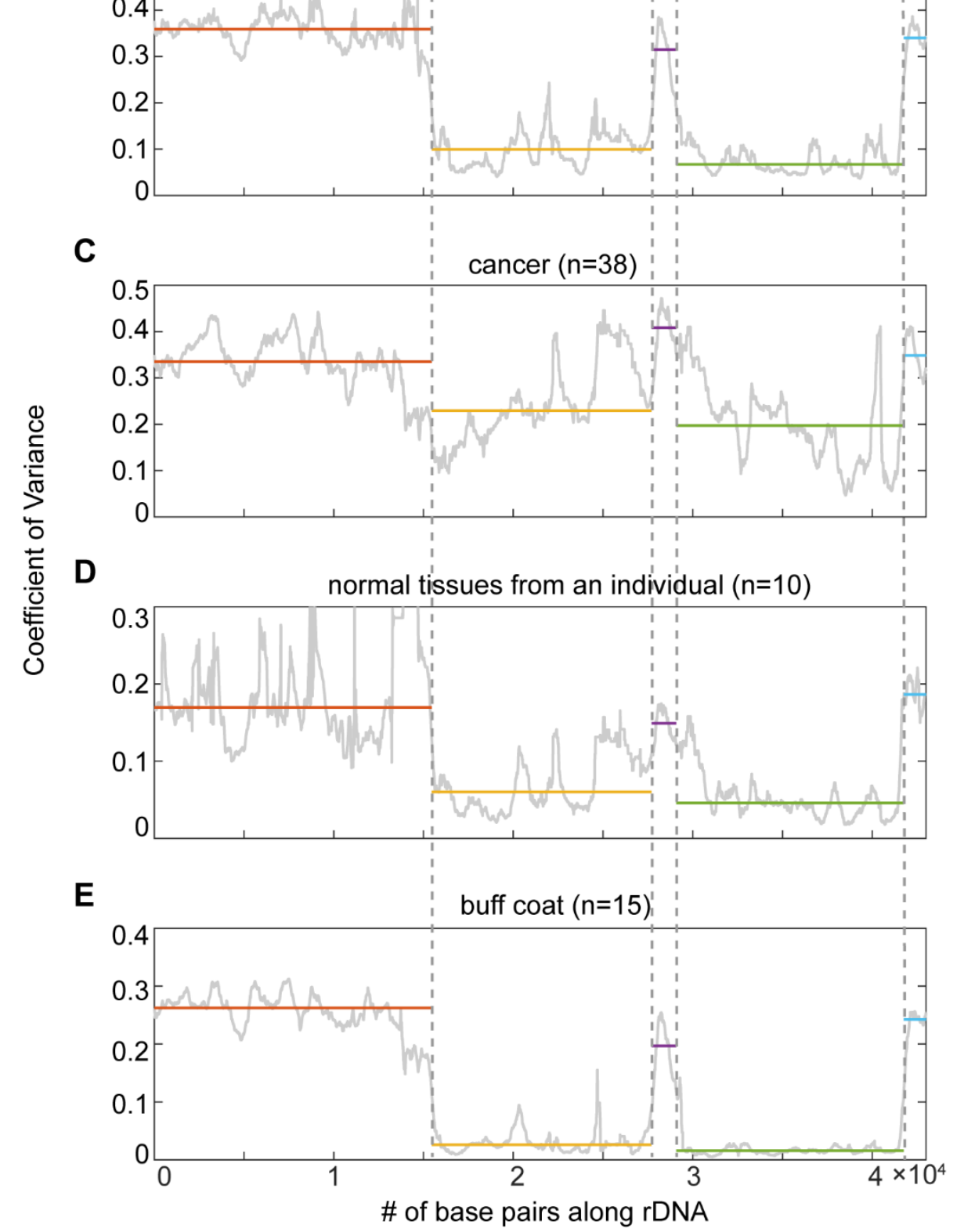

Supplementary Figure 1 Methylation variations across tumors, normal tissues and individuals

(A) A schematic representation of human $r D N A$ repeat unit. (B) Plot of coefficient of variance

(C.V.) of normal tissues in rDNA. For each zone, median of C.V. is drew as horizontal line. (C)

Plot of C.V. of tumors on rDNA. (D) Plot of C.V. of ten adult tissues from the same individual on rDNA. (E) Plot of C.V. of buff coats from 15 individuals. 
A
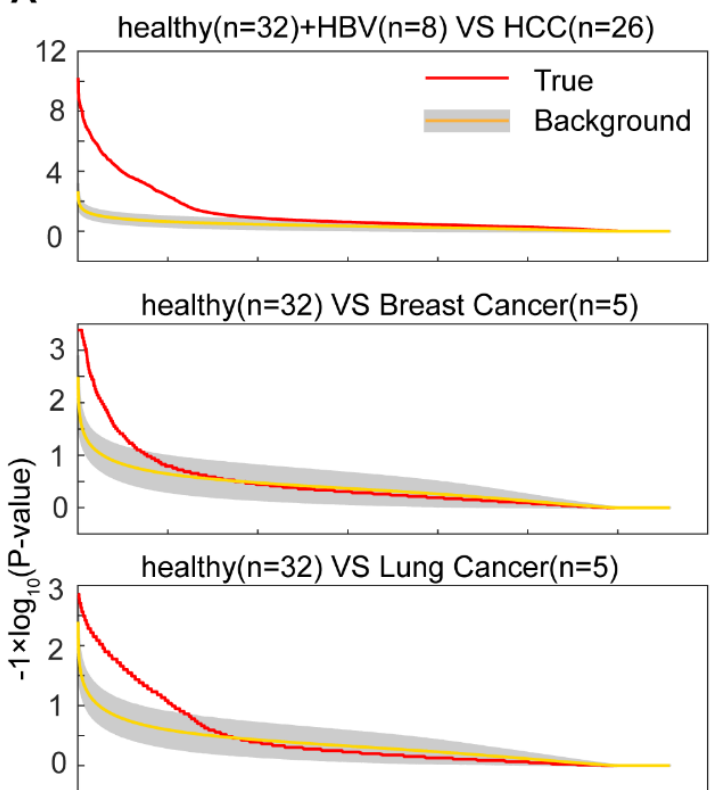

healthy $(n=32)$ VS Nasopharyngeal Cancer $(n=9)$

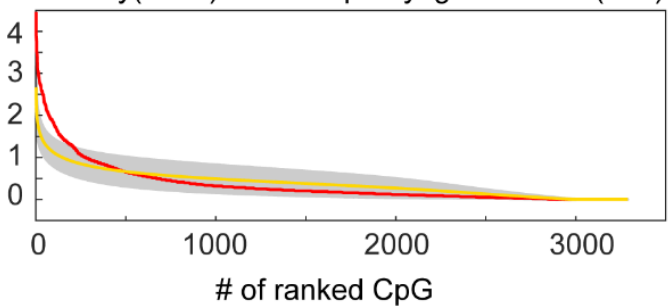

B
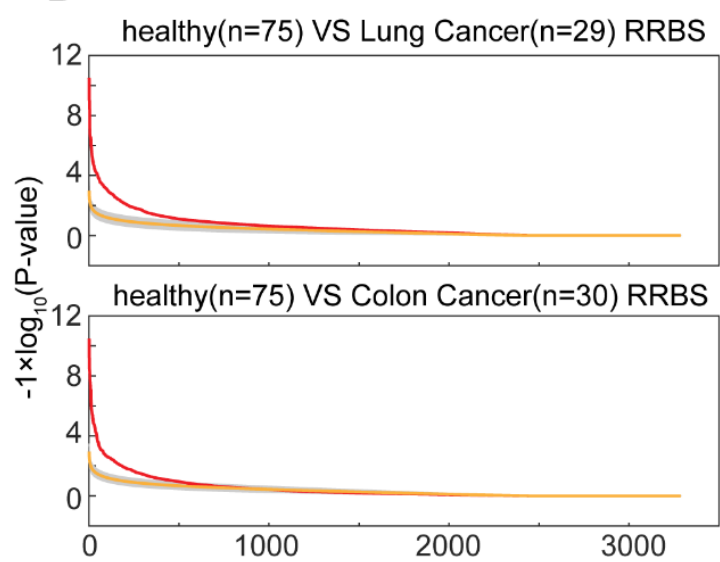

\# of ranked CpG

Supplementary Figure 2 Significance of differences between healthy and cancer plasma (A) Plots of significance of differences between healthy subjects and cancer patients with WGBS data. $C p G$ dinucleotides are ranked by the P-values of Wilcoxon rank sum test. Red solid lines show the real results; yellow solid lines with grey covers show the results of 100 times'shuffling. Grey covers shows the intervals of \pm 1 standard derivation. (B) Plots of significance of differences between healthy subjects and cancer patients with RRBS data. 
A

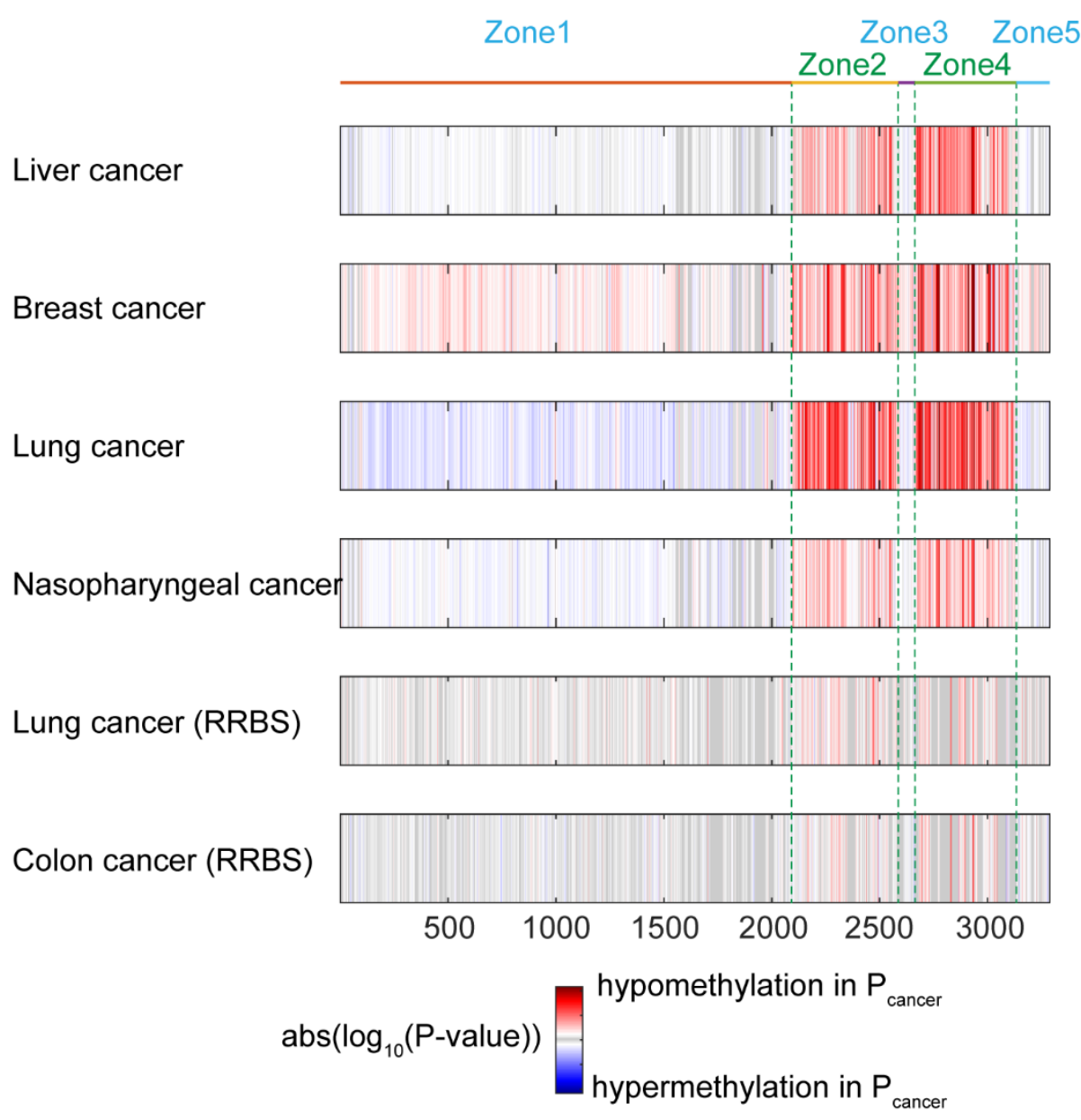

\section{Supplementary Figure 3 Differential CpG sites between healthy plasma and cancer plasma}

(A) Heat maps of significance of differences distinguishing healthy and cancer patients on single $C p G$ dinucleotides. $C p G$ dinucleotides are ranked by positions on $r D N A$. The maximum of red or blue color indicates the most significant $C p G$ 's absolute log10(P-value) no matter it is either hypo-methylation or hyper-methylation. On the heat maps, we found that almost all of significant CpG sites show hypo-methylation. 
A

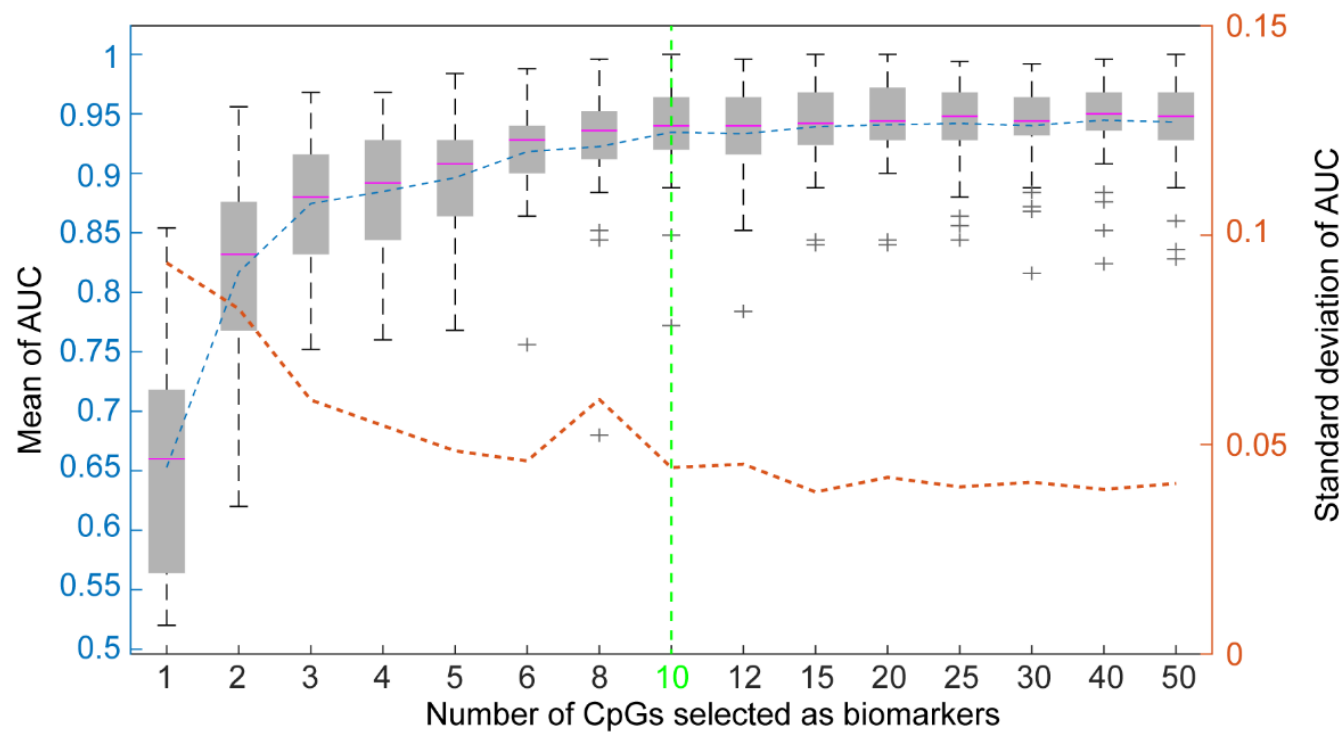

Supplementary Figure 4 Performances in distinguishing healthy and lung cancer plasma with different numbers of CpG sites (RRBS)

(A) Boxplots of AUCs of SVM classifier with different numbers of CpG sites. Blue dashed line shows mean AUCs, while red dashed line shows AUCs'standard derivation. 


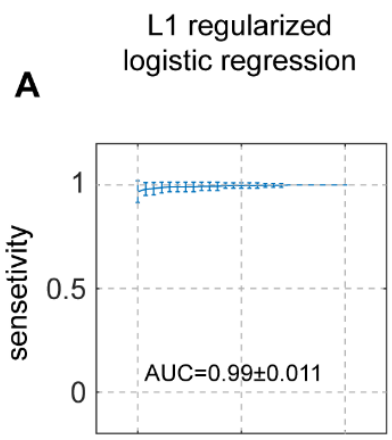

$\mathbf{B}$

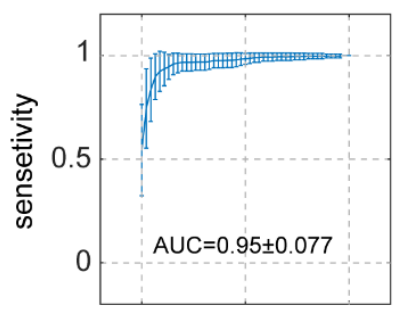

C

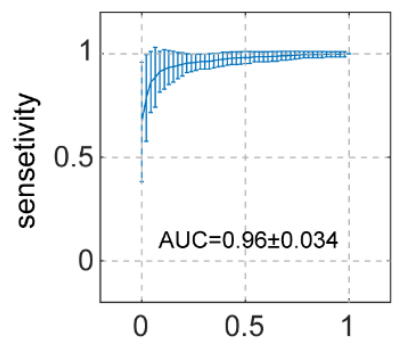

SVM

Liver cancer (WGBS)

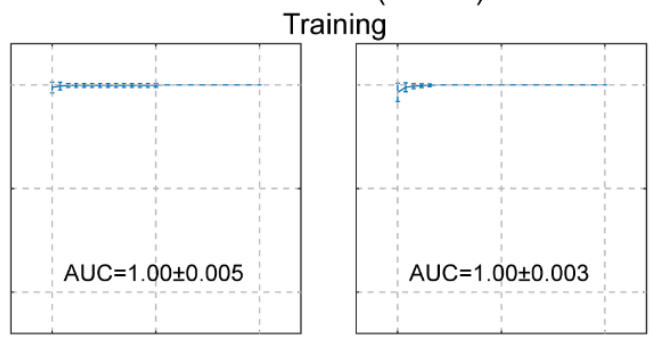

Lung cancer (RRBS)

Training
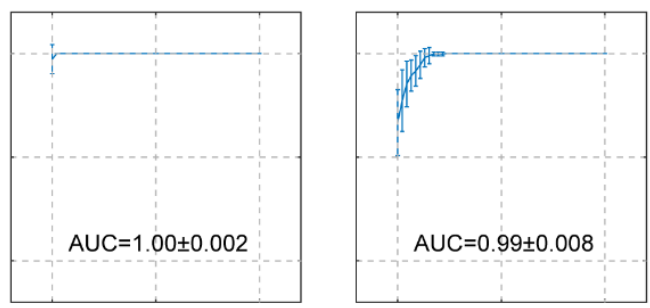

Colon cancer (RRBS)

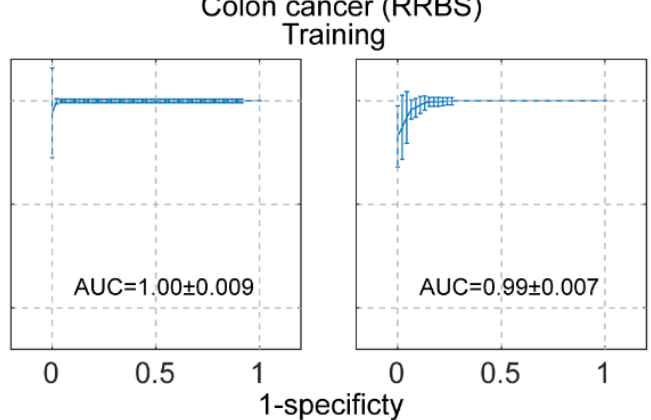

Random forest
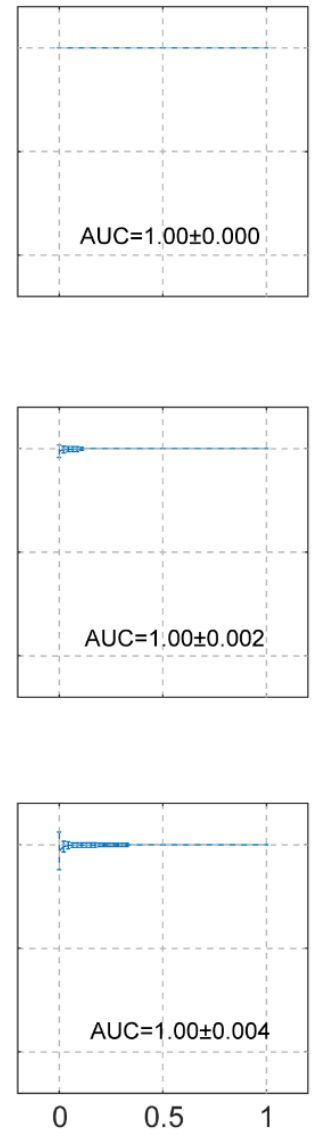

Supplementary Figure 5 Prediction performances of training dataset.

(A) Training ROC curves for liver cancer plasma and healthy plasma with four classifiers (WGBS). Standard derivation bars are labeled showing the performance variation of 50 runs. AUCs are labeled on the plots. (B) Training ROC curves for lung cancer plasma and healthy plasma with four models (RRBS). (C) Training ROC curves for colon cancer plasma and healthy plasma with four models (RRBS). 
bioRxiv preprint doi: https://doi org/10.1101/651497; this version posted May 29 2019. The copyright holder for this preprint (which was not certified by peer review) is the author/funder, who has granted bioRxiv a license to display the preprint in perpetuity. It is made available under aCC-BY-NC-ND 4.0 International license.

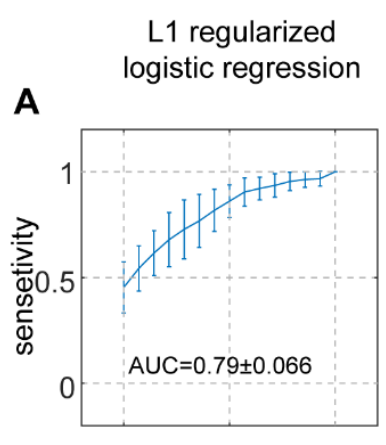

SVM

KNN

Testing on other cancer types (WGBS)
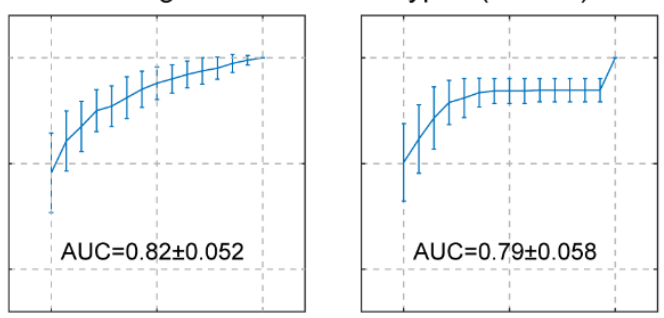

Testing on colon cancer (RRBS)

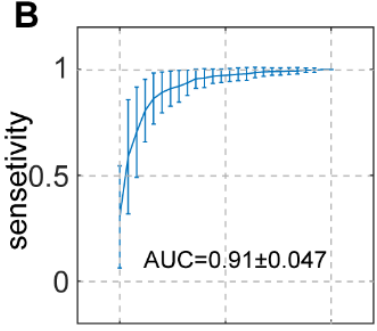

C

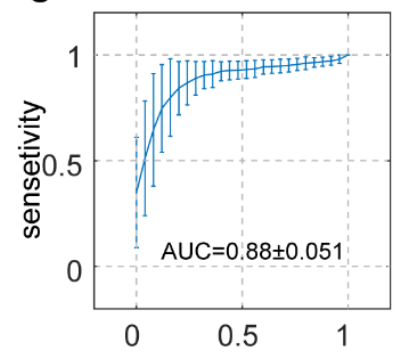

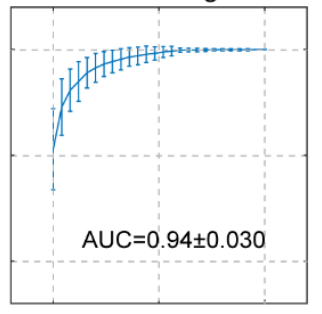

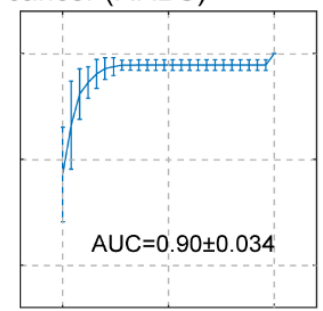

Testing on lung cancer (RRBS)

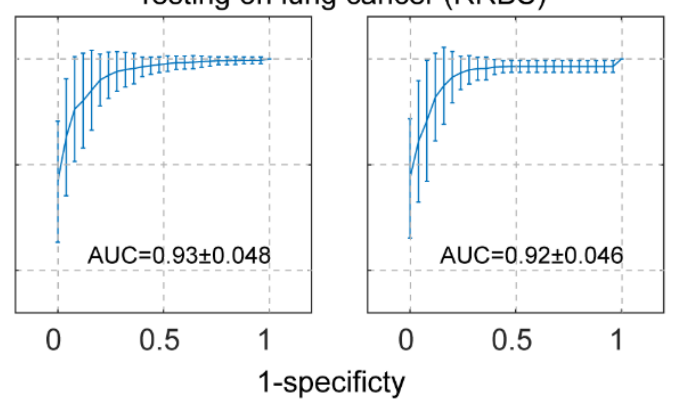

Random forest
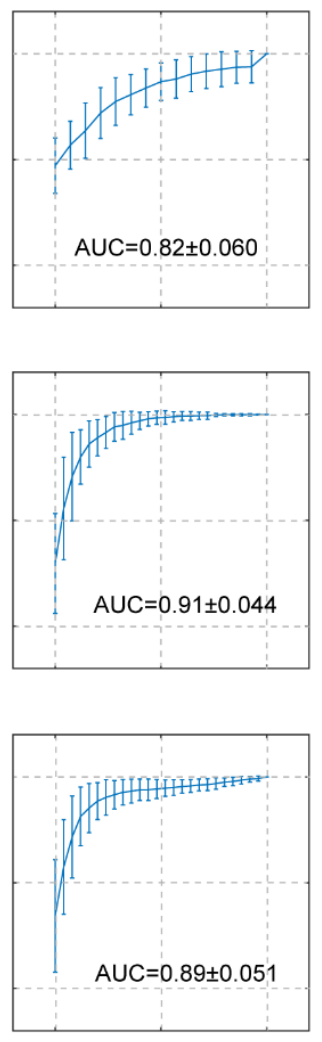

$\begin{array}{lll}0 & 0.5 & 1\end{array}$

Supplementary Figure 6 Prediction performances of other cancer types.

(A) Testing ROC curves for other cancer types (including breast cancer, lung cancer and nasopharyngeal cancer) plasma WGBS data with four classifiers trained with liver cancer plasma WGBS. Standard derivation bars are labeled showing the performance variation of 50 runs. AUCs are labeled on the plots. (B) Testing ROC curves for colon cancer plasma RRBS with four classifiers trained with lung cancer plasma RRBS. (C) Testing ROC curves for lung cancer plasma RRBS with four classifiers trained with colon cancer plasma RRBS. 
L1 regularized logistic regression

A
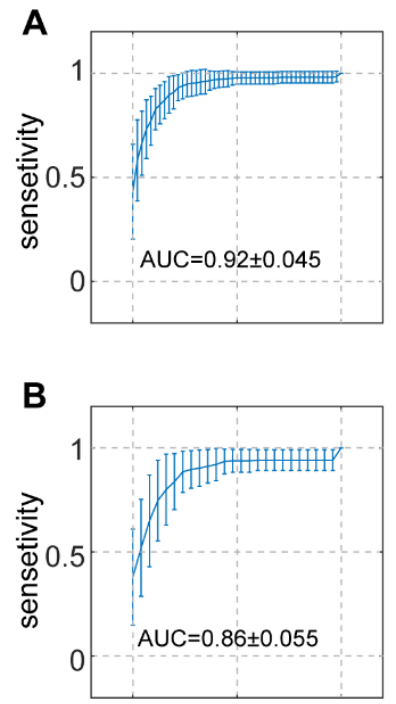

C

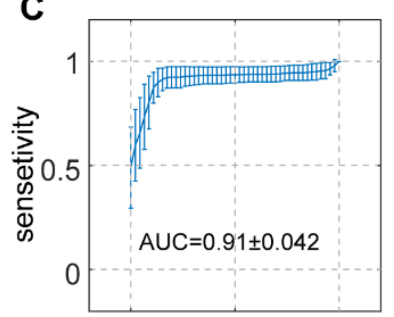

D

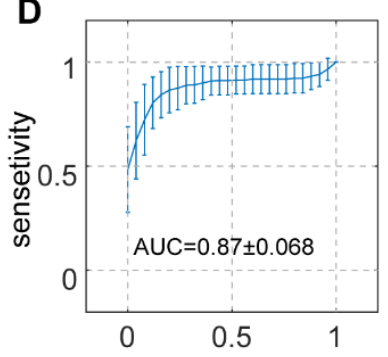

SVM

KNN

Random forest

Lung cancer RRBS with markers from Liver cancer WGBS
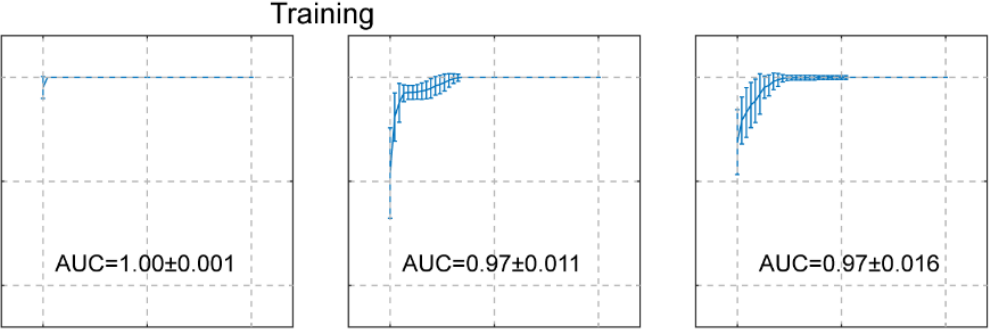

Testing
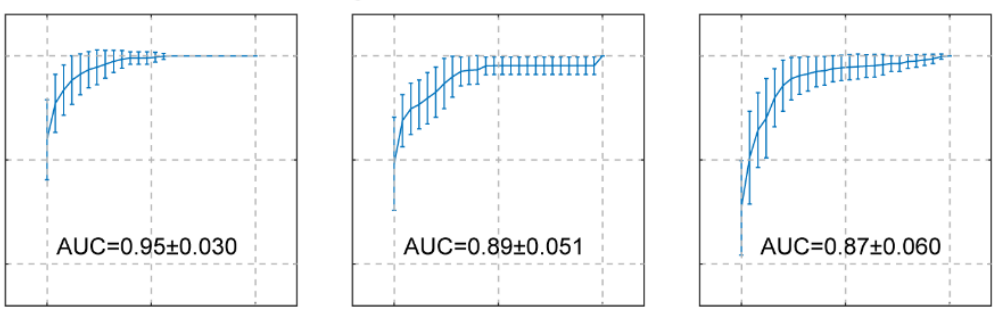

Colon cancer RRBS with markers from Liver cancer WGBS Training
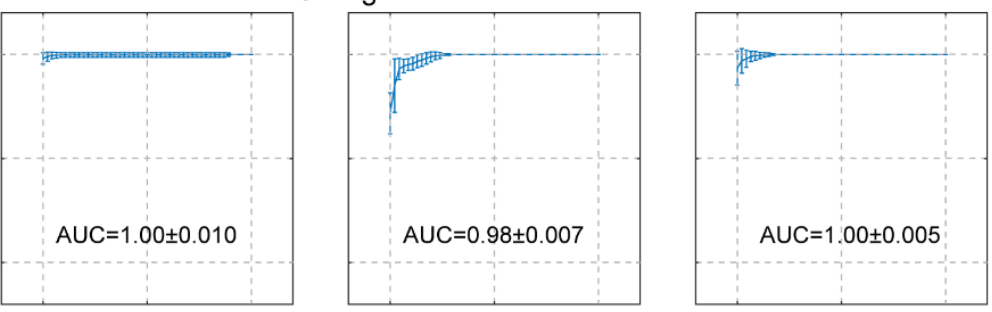

Testing
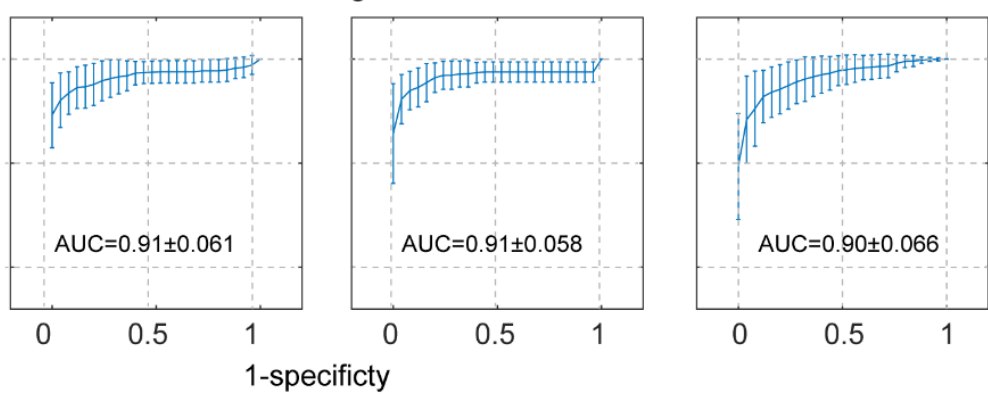

Supplementary Figure 7 Prediction performances of RRBS plasma with CpG sites transferred from liver cancer WGBS dataset.

(A) Training ROC curves for lung cancer plasma RRBS with CpG sites selected in liver cancer $W G B S$ dataset. Standard derivation bars are labeled showing the performance variation of 50 runs. AUCs are labeled on the plots. (B) Testing ROC curves for lung cancer plasma RRBS with $C p G$ sites selected in liver cancer WGBS dataset. (C) Training ROC curves for colon cancer plasma RRBS with CpG sites selected in liver cancer WGBS dataset. (D) Testing ROC curves for colon cancer plasma RRBS with CpG sites selected in liver cancer WGBS dataset. 
bioRxiv preprint doi: https://doi org/101101/651497; this version posted May 29, 2019. The copyright holder for this preprint (which was not certified by peer review) is the author/funder, who has granted bioRxiv a license to display the preprint in perpetuity. It is made available under aCC-BY-NC-ND 4.0 International license.

L1 regularized logistic regression

A

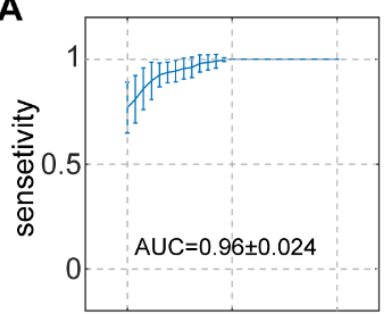

B
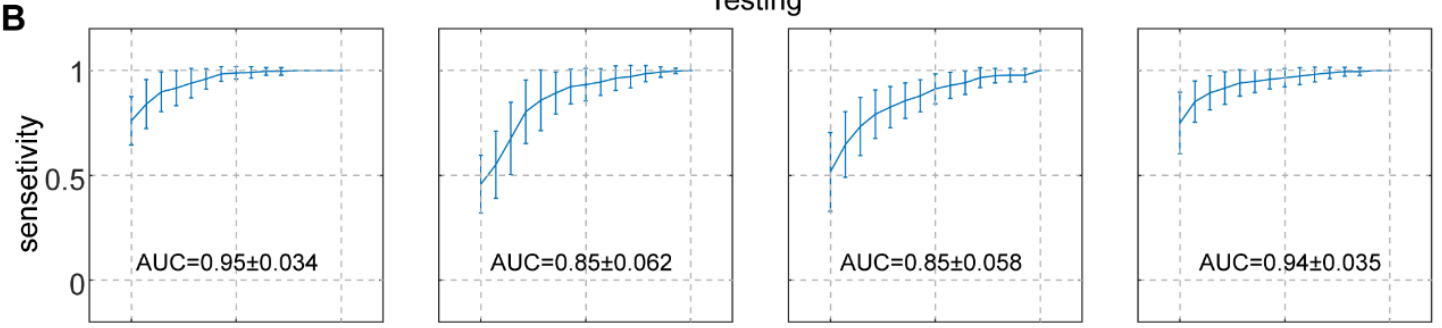

Liver cancer WGBS with markers from Colon cancer WGBS

C

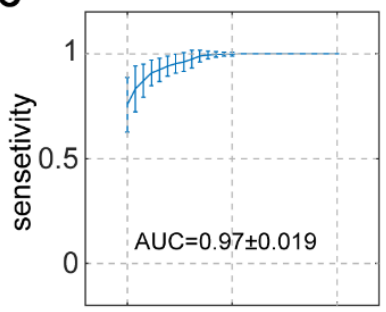

D

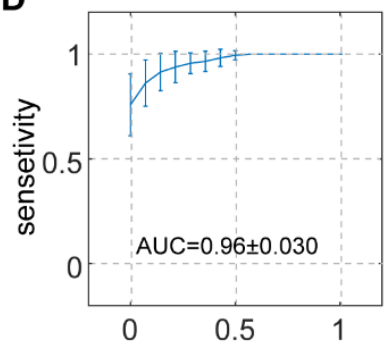

Testing

SVM

KNN

Random forest

Liver cancer WGBS with markers from Lung cancer RRBS
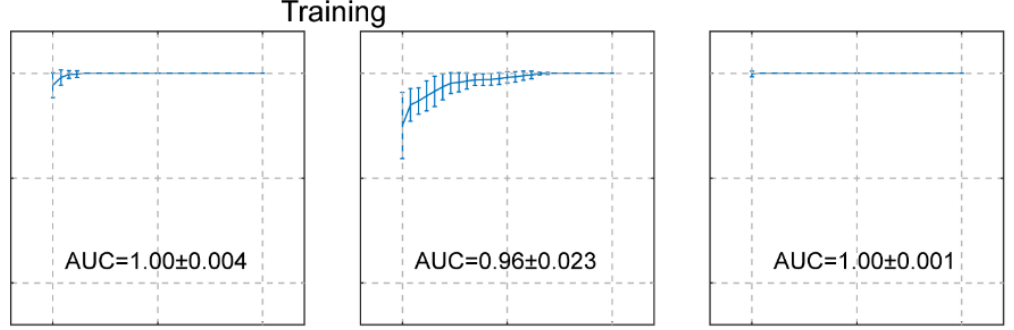

$\mathrm{AUC}=1.00 \pm 0.001$

AUC $=0.94 \pm 0.035$

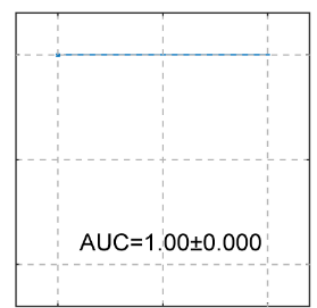

Testing
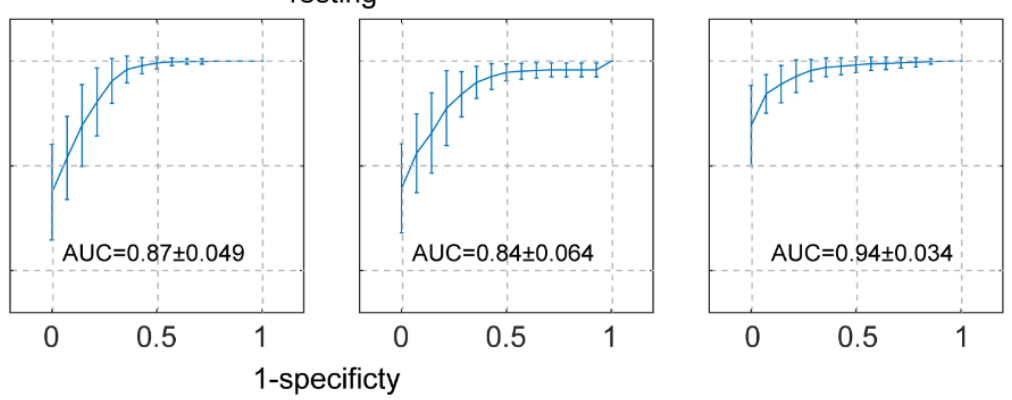

Supplementary Figure 8 Prediction performances of liver cancer plasma WGBS with CpG sites transferred from RRBS dataset.

(A) Training ROC curves for liver cancer plasma WGBS with CpG sites selected in lung cancer plasma RRBS dataset. Standard derivation bars are labeled showing the performance variations of 50 runs. AUCs are labeled on the plots. (B) Testing ROC curves for liver cancer plasma WGBS with CPG sites selected in lung cancer plasma RRBS dataset. (C) Training ROC curves for liver cancer plasma WGBS with Cp G sites selected in colon cancer plasma RRBS dataset. (D) Testing ROC curves for liver cancer plasma WGBS with CpG sites selected in colon cancer plasma RRBS dataset. 

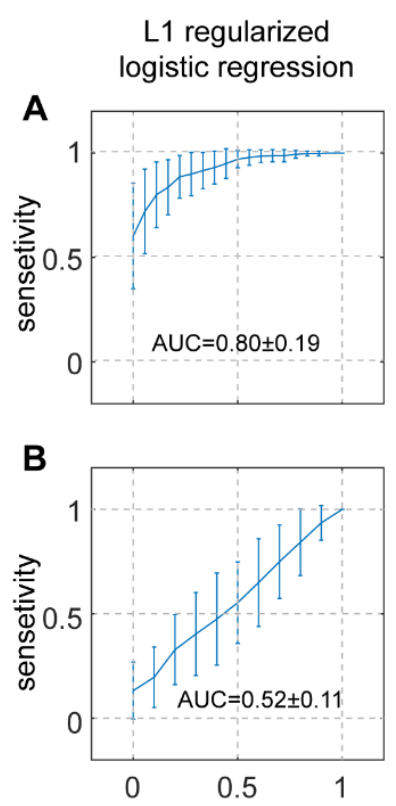

SVM

Training to distinguish two cancers

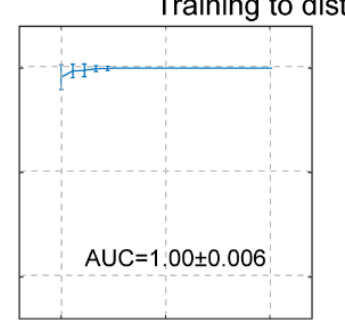

Testing to distinguish two cancers

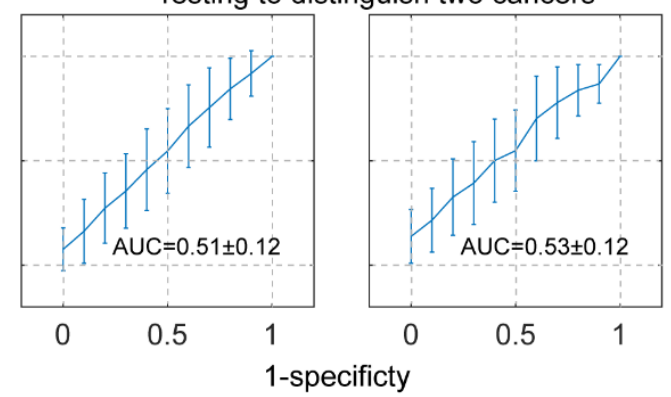

Random forest
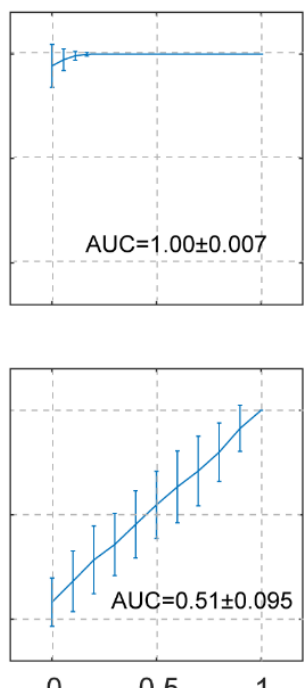

Supplementary Figure 9 Performances of distinguishing lung cancer and colon cancer plasma RRBS.

(A) Training ROC curves for distinguishing lung cancer plasma and colon cancer plasma (RRBS). Standard derivation bars are labeled showing the performance variations of 50 runs. AUCs are labeled on the plots. (B) Testing ROC curves for distinguishing lung cancer plasma and colon cancer plasma (RRBS). 
[1] Chan KC, Jiang P, Chan CW, Sun K, Wong J, Hui EP, Chan SL, Chan WC, Hui DS, Ng SS et al: Noninvasive detection of cancer-associated genome-wide hypomethylation and copy number aberrations by plasma DNA bisulfite sequencing. Proc Natl Acad Sci U S A 2013, 110(47):1876118768 .

[2] Guo S, Diep D, Plongthongkum N, Fung HL, Zhang K, Zhang K: Identification of methylation haplotype blocks aids in deconvolution of heterogeneous tissue samples and tumor tissue-of-origin mapping from plasma DNA. Nat Genet 2017, 49(4):635-642.

[3] Li X, Liu Y, Salz T, Hansen KD, Feinberg A: Whole-genome analysis of the methylome and hydroxymethylome in normal and malignant lung and liver. Genome Res 2016, 26(12):1730-1741. [4] Heyn H, Vidal E, Ferreira HJ, Vizoso M, Sayols S, Gomez A, Moran S, Boque-Sastre R, Guil S, Martinez-Cardus A et al: Epigenomic analysis detects aberrant super-enhancer DNA methylation in human cancer. Genome Biol 2016, 17:11.

[5] GSE104789, https://www.ncbi.nlm.nih.gov/geo/query/acc.cgi 